\title{
Total Synthesis of the Sensitive Triyne Natural Product (4S,5S)-4,8- Dihydroxy-3,4-dihydrovernoniyne and all its Stereoisomers
}

Gujjula. V. Ramakrishna and Rodney A. Fernandes*

\section{Table of contents}

General information $\quad$ S2

Procedure for synthesis and characterization data of compounds 14, 18, and $13 \quad$ S2-S4

Procedure for synthesis and characterization data for compounds 20, 21, and $12 \quad S 4-S 5$

Procedure for synthesis and characterization data for compounds 22 and $\mathbf{1 b}$

Procedure for synthesis and characterization data of compounds $16,23,24,25$, and 1a S6-S8

Procedures for synthesis and characterization data of compounds ent-14, ent-18, ent-13, ent-22, and ent-1b

S8-S9

Procedures for synthesis and characterization data of compounds ent-16, ent-23, ent-24, ent-25, and ent-1a

S9-S10

References $\quad S 10$

${ }^{1} \mathrm{H}$ and ${ }^{13} \mathrm{C}$ spectra of all compounds $\quad$ S11-S25 


\section{General Information}

Solvents and reagents were purified by standard methods. Thin-layer chromatography was performed on EM 250 Kieselgel 60 F254 silica gel plates. The spots were visualized by staining with $\mathrm{KMnO}_{4}$ or under a UV lamp. ${ }^{1} \mathrm{H}$ and ${ }^{13} \mathrm{C}$ NMR (proton decoupled) were recorded with a spectrometer operating at 500 or 400 and 125 or $100 \mathrm{MHz}$ for proton and carbon nuclei respectively. The chemical shifts are based on the TMS peak at $\delta=0.00 \mathrm{ppm}$ for proton NMR and the $\mathrm{CDCl}_{3}$ peak at $\delta=77.00 \mathrm{ppm}(\mathrm{t})$, acetone- $\mathrm{d}_{6}$ peak at 29.92 (s) in carbon NMR. IR spectra were obtained on an FT-IR spectrometer, and the samples were prepared by evaporation from $\mathrm{CHCl}_{3}$ on $\mathrm{CsBr}$ plates. High-resolution mass spectra (HRMS) were obtained using positive electrospray ionization by the TOF method.

\section{$(4 S, 5 S)-4-H y d r o x y-5$-vinyl-dihydrofuran-2(3H)-one $(14)^{1}$}

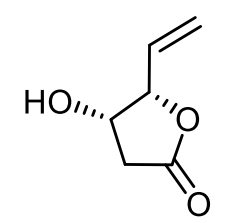

To L-mannonic- $\gamma$-lactone 15 (4.5 g, $25.26 \mathrm{mmol})$ was added 33\% hydrogen bromide in acetic acid (HBA, $18 \mathrm{~mL}$ ) and then the reaction mixture was stirred at $50{ }^{\circ} \mathrm{C}$ for $1 \mathrm{~h}$. Excess HBA was removed under reduced pressure. The resultant syrupy liquid was dissolved in $50 \%$ aqueous acetic acid $(45 \mathrm{~mL})$, then cooled to $-10{ }^{\circ} \mathrm{C}$, and zinc powder (9.08 g, $138.93 \mathrm{mmol}, 5.5$ equiv) was added in portions over $2 \mathrm{~h}$ at $-10^{\circ} \mathrm{C}$. The mixture was stirred and warmed to room temperature over $2 \mathrm{~h}$ and then heated at $60{ }^{\circ} \mathrm{C}$ for an additional $1 \mathrm{~h}$. It was then filtered and the filtrate concentrated under reduced pressure. The residue was dissolved in water (35 $\mathrm{mL}$ ) and cooled to $0{ }^{\circ} \mathrm{C}$ and $\mathrm{pH}$ adjusted to 10 by slow addition of $\mathrm{KOH}$ pellets to precipitate the remaining zinc as the insoluble hydroxide and to effect C-3 deacetylation. After filtration, the basic filtrate was acidified to $\mathrm{pH} 5$ using hydrochloric acid at $0{ }^{\circ} \mathrm{C}$. Water was removed under reduced pressure and the residue dissolved in cold ethanol. Precipitated potassium chloride was removed by filtration and the filtrate concentrated. The residue was purified by column chromatography using petroleum ether/EtOAc $(1: 1)$ as eluent to give $14(1.55 \mathrm{~g}, 48 \%)$ as pale yellow oil. $[\alpha]_{\mathrm{D}}{ }^{25}=-45.8(c=$ 1.0, $\left.\mathrm{CHCl}_{3}\right)$; IR $\left(\mathrm{CHCl}_{3}\right): v_{\max }=3444,2928,1772,1648,1431,1332,1157,1081,1018,939,900,833$, 706, $668 \mathrm{~cm}^{-1} ;{ }^{1} \mathrm{H}$ NMR (500 MHz, $\left.\mathrm{CDCl}_{3}\right): \delta=5.98-5.90(\mathrm{~m}, 1 \mathrm{H}), 5.49(\mathrm{~d}, J=17.5 \mathrm{~Hz}, 1 \mathrm{H}), 5.43(\mathrm{~d}$, $J=11.0 \mathrm{~Hz}, 1 \mathrm{H}), 4.86(\mathrm{t}, J=4.7 \mathrm{~Hz}, 1 \mathrm{H}), 4.50(\mathrm{t}, J=4.0 \mathrm{~Hz}, 1 \mathrm{H}), 3.20(\mathrm{br} \mathrm{s}, 1 \mathrm{H}, O H), 2.76(\mathrm{dd}, J=$ 17.6, $5.6 \mathrm{~Hz}, 1 \mathrm{H}), 2.55(\mathrm{dd}, J=17.6,5.6 \mathrm{~Hz}, 1 \mathrm{H}) \mathrm{ppm} ;{ }^{13} \mathrm{C}\left\{{ }^{1} \mathrm{H}\right\} \mathrm{NMR}\left(125 \mathrm{MHz}, \mathrm{CDCl}_{3}\right): \delta=176.1$, 130.2, 120.6, 84.9, 69.4, 38.6 ppm; HRMS (ESI-TOF) $m / z:[\mathrm{M}+\mathrm{Na}]^{+}$Calcd for $\mathrm{C}_{6} \mathrm{H}_{8} \mathrm{O}_{3} \mathrm{Na}_{151.0366}$; Found 151.0368. 


\section{(4S,5S)-4-(tert-Butyldimethylsilyloxy)-5-vinyldihydrofuran-2(3H)-one (18)}

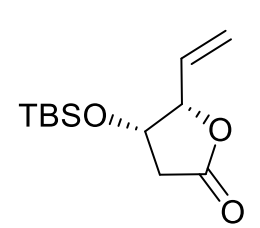

To a solution of hydroxy lactone $14(0.5 \mathrm{~g}, 3.903 \mathrm{mmol})$ in dry $\mathrm{CH}_{2} \mathrm{Cl}_{2}(15 \mathrm{~mL})$ was added imidazole $(0.531 \mathrm{~g}, 7.805 \mathrm{mmol}, 2.0$ equiv), followed by tertbutyldimethylsilyl chloride $(0.882 \mathrm{~g}, 5.855 \mathrm{mmol}, 1.5$ equiv $)$ at $0{ }^{\circ} \mathrm{C}$. The reaction mixture was stirred at room temperature for $12 \mathrm{~h}$ and then diluted with $\mathrm{CH}_{2} \mathrm{Cl}_{2}$ (10 mL) and $\mathrm{H}_{2} \mathrm{O}(10 \mathrm{~mL})$. The solution was extracted with $\mathrm{CH}_{2} \mathrm{Cl}_{2}(3 \times 20 \mathrm{~mL})$. The combined organic phases were washed with water, brine, dried $\left(\mathrm{Na}_{2} \mathrm{SO}_{4}\right)$, and concentrated. The residue was purified by silica gel column chromatography using petroleum ether/EtOAc (9:1) as eluent to afford $18(0.870 \mathrm{~g}, 92 \%)$ as colorless oil. $[\alpha]_{\mathrm{D}}{ }^{25}=-16.5\left(c=1.0, \mathrm{CHCl}_{3}\right)$. IR $\left(\mathrm{CHCl}_{3}\right): v_{\max }=2956$, 2932, 2859,1781, 1473, 1259, 1206, 1149, 1096, 1029, 946, 841, $779 \mathrm{~cm}^{-1}$; ${ }^{1} \mathrm{H}$ NMR (400 MHz, $\left.\mathrm{CDCl}_{3}\right): \delta=5.97(\mathrm{ddd}, J=17.4,10.4,7.2 \mathrm{~Hz}, 1 \mathrm{H}), 5.42(\mathrm{dt}, J=17.4,1.1 \mathrm{~Hz}, 1 \mathrm{H}), 5.37(\mathrm{dt}, J=$ $10.5,1.0 \mathrm{~Hz}, 1 \mathrm{H}), 4.79(\mathrm{dd}, J=7.2,4.2 \mathrm{~Hz}, 1 \mathrm{H}), 4.51-4.48(\mathrm{~m}, 1 \mathrm{H}), 2.73(\mathrm{dd}, J=17.2,5.4 \mathrm{~Hz}$, $1 \mathrm{H}), 2.48(\mathrm{dd}, J=17.2,2.1 \mathrm{~Hz}, 1 \mathrm{H}), 0.86(\mathrm{~s}, 9 \mathrm{H}), 0.04(\mathrm{~s}, 3 \mathrm{H}), 0.03(\mathrm{~s}, 3 \mathrm{H}) \mathrm{ppm} ;{ }^{13} \mathrm{C}\left\{{ }^{1} \mathrm{H}\right\} \mathrm{NMR}$ $\left(100 \mathrm{MHz}_{\mathrm{CDCl}}\right): \delta=175.2,131.8,119.9,85.4,70.7,39.2,25.6,18.0,-4.9,-5.0$ ppm; HRMS (ESI-TOF) $m / z:[\mathrm{M}+\mathrm{H}]^{+}$Calcd for $\mathrm{C}_{12} \mathrm{H}_{23} \mathrm{O}_{3} \mathrm{Si}$ 243.1415; Found 243.1418.

\section{(4S,5S)-4-(tert-Butyldimethylsilyloxy)-5-(prop-2-yn-1-yl)dihydrofuran-2(3H)-one (13)}

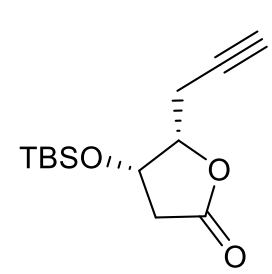

A mixture of $\mathrm{PdCl}_{2}(30.7 \mathrm{mg}, 0.173 \mathrm{mmol}, 20 \mathrm{~mol} \%)$ and $\mathrm{CuCl}(129 \mathrm{mg}, 1.3$ mmol, 1.5 equiv) in DMF $(7 \mathrm{~mL})$ and $\mathrm{H}_{2} \mathrm{O}(1 \mathrm{~mL})$ was stirred for 20 min at room temperature. To this mixture was added compound 18 (210 mg, 0.866

mmol) in DMF (2 mL) and oxygen gas was bubbled through the solution for 8 h. It was then filtered through a small pad of silica gel and washed with EtOAc. The filtrate was washed with water $(3 \times 50 \mathrm{~mL})$, brine, dried $\left(\mathrm{Na}_{2} \mathrm{SO}_{4}\right)$ and concentrated to give the crude aldehyde (200 mg) as a colorless oil, which was used directly in the next reaction. To a solution of diethyl (diazomethyl)phosphonate $\mathbf{Z}^{2}$ (308 mg, $1.732 \mathrm{mmol}, 2.0$ equiv) in THF ( $3 \mathrm{~mL}$ ), cooled to $-78^{\circ} \mathrm{C}$ under $\mathrm{N}_{2}$ was added $t$-BuOK (194 mg, $1.732 \mathrm{mmol}, 2.0$ equiv). The reaction mixture was stirred at $-78{ }^{\circ} \mathrm{C}$ for $15 \mathrm{~min}$ and then a solution of the above aldehyde $(0.200 \mathrm{~g})$ in THF $(2 \mathrm{~mL})$ was added dropwise over $1 \mathrm{~h}$ and the mixture stirred for further $1 \mathrm{~h}$. It was then quenched with a saturated aq. $\mathrm{NH}_{4} \mathrm{Cl}$ solution, and extracted with EtOAc $(3 \times 30 \mathrm{~mL})$. The combined organic layers were washed with brine, dried $\left(\mathrm{Na}_{2} \mathrm{SO}_{4}\right)$ and concentrated. The residue was purified by silica gel column chromatography using petroleum ether/EtOAc (4:1) as eluent to give the alkyne 13 (121 $\mathrm{mg}, 55 \%)$ as yellow solid, M.p. $55-58{ }^{\circ} \mathrm{C} .[\alpha]_{\mathrm{D}}{ }^{25}=+4.3\left(c=1.0, \mathrm{CHCl}_{3}\right)$; IR $\left(\mathrm{CHCl}_{3}\right): v_{\max }=3302,2930$, 
2253, 1781, 1620, 1416, 1350, 1193, 1159, 1070, 1031, 987, 911, 736, $649 \mathrm{~cm}^{-1} ;{ }^{1} \mathrm{H}$ NMR (400 $\left.\mathrm{MHz}_{\mathrm{CDCl}}\right): \delta=4.54(\mathrm{dt}, J=8.6,4.8 \mathrm{~Hz}, 1 \mathrm{H}), 4.50-4.46(\mathrm{~m}, 1 \mathrm{H}), 2.75-2.68(\mathrm{~m}, 3 \mathrm{H}), 2.48(\mathrm{dd}$, $J=17.3,1.0 \mathrm{~Hz}, 1 \mathrm{H}), 2.0(\mathrm{t}, J=2.6 \mathrm{~Hz}, 1 \mathrm{H}), 0.89(\mathrm{~s}, 9 \mathrm{H}), 0.12(\mathrm{~s}, 3 \mathrm{H}), 0.10(\mathrm{~s}, 3 \mathrm{H}) \mathrm{ppm} ;{ }^{13} \mathrm{C}\left\{{ }^{1} \mathrm{H}\right\}$ NMR (100 MHz, $\left.\mathrm{CHCl}_{3}\right): \delta=174.7,82.3,78.9,70.7,68.6,39.5,25.6,18.6,17.9,-4.7,-5.3$ ppm; HRMS (ESI-TOF) $m / z:[\mathrm{M}+\mathrm{Na}]^{+}$Calcd for $\mathrm{C}_{13} \mathrm{H}_{22} \mathrm{O}_{3} \mathrm{SiNa} 277.1230$; Found 277.1231.

\section{5-Trimethylsilyl-penta-2,4-diyn-1-ol (20) ${ }^{3}$}

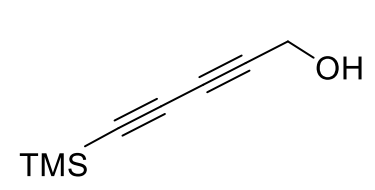

To a stirred solution of propargyl alcohol 19 (2.3 mL, $40 \mathrm{mmol}, 2.0$ equiv) and trimethylsilylacetylene $(2.8 \mathrm{~mL}, 20 \mathrm{mmol}, 1.0$ equiv) in acetone (60 $\mathrm{mL})$ at room temperature were added copper(I) iodide $(0.762 \mathrm{~g}, 4 \mathrm{mmol}$, $20 \mathrm{~mol} \mathrm{\%}$ ) and tetramethylethylenediamine (TMEDA, $0.930 \mathrm{~g}, 8 \mathrm{mmol}, 40 \mathrm{~mol} \%$ ) under $\mathrm{O}_{2}$. The reaction mixture was stirred at room temperature for $8 \mathrm{~h}$. After completion of reaction, water (15 $\mathrm{mL})$ was added and the mixture extracted with ether $(3 \times 50 \mathrm{~mL})$. The combined organic layers were dried $\left(\mathrm{Na}_{2} \mathrm{SO}_{4}\right)$, filtered, and concentrated under reduced pressure. The residue was purified by flash column chromatography using hexane/EtOAc (19:1) to give $\mathbf{2 0}(2.38 \mathrm{~g}, 78 \%)$ as colorless oil. ${ }^{1} \mathrm{H}$ NMR (500 MHz, $\left.\mathrm{CDCl}_{3}\right): \delta=4.3(\mathrm{~s}, 2 \mathrm{H}), 0.19$ (s, 9H) ppm; ${ }^{13} \mathrm{C}\left\{{ }^{1} \mathrm{H}\right\} \mathrm{NMR}(125 \mathrm{MHz}$, $\left.\mathrm{CDCl}_{3}\right): \delta=87.8,87.1,75.8,70.7,51.4,-0.5 \mathrm{ppm}$.

\section{Penta-2,4-diyn-1-ol (21)}

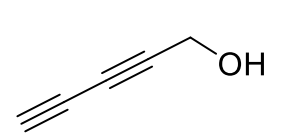

To a stirred solution of alcohol 20 (1.0 g, $6.56 \mathrm{mmol})$ in $\mathrm{MeOH}(15 \mathrm{~mL})$ was added $\mathrm{K}_{2} \mathrm{CO}_{3}$ (1.36 g, $9.85 \mathrm{mmol}, 1.5$ equiv). The reaction mixture was stirred for $1 \mathrm{~h}$ at room temperature and then quenched with a saturated aq. solution of $\mathrm{NH}_{4} \mathrm{Cl}(20 \mathrm{~mL})$, and extracted with $\mathrm{Et}_{2} \mathrm{O}(3 \times 50 \mathrm{~mL})$. The combined organic extracts were washed with brine, dried $\left(\mathrm{Na}_{2} \mathrm{SO}_{4}\right)$ and concentrated. The crude product was purified by silica gel column chromatography using petroleum ether/EtOAc (9:1) to afford diyne alcohol $21(0.473 \mathrm{~g}, 90 \%)$ as pale yellow oil. ${ }^{1} \mathrm{H}$ NMR (400 MHz, $\left.\mathrm{CDCl}_{3}\right): \delta=4.32$ (s, 2H), $2.19(\mathrm{~s}, 1 \mathrm{H}) \mathrm{ppm} ;{ }^{13} \mathrm{C}\left\{{ }^{1} \mathrm{H}\right\} \mathrm{NMR}$ $\left(100 \mathrm{MHz}, \mathrm{CDCl}_{3}\right): \delta=74.4,70.0,68.5,67.4,51.3 \mathrm{ppm}$.

\section{5-Bromopenta-2,4-diyn-1-ol (12)}

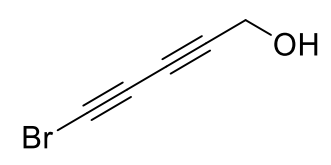

To a stirred solution of alkyne 21 (100 mg, $1.25 \mathrm{mmol})$ in acetone (4 mL), was added NBS (246 mg, $1.38 \mathrm{mmol}, 1.1$ equiv) and silver nitrate (21.2 mg, $0.125 \mathrm{mmol}, 10 \mathrm{~mol} \%)$ under nitrogen atmosphere at $0{ }^{\circ} \mathrm{C}$. The reaction mixture was stirred at $0{ }^{\circ} \mathrm{C}$ for $1 \mathrm{~h}$. After completion of the reaction (monitored by TLC), the mixture was cooled to $0{ }^{\circ} \mathrm{C}$ and quenched with cold water $(1 \mathrm{~mL})$. The reaction mixture was 
extracted with $\mathrm{Et}_{2} \mathrm{O}(3 \times 10 \mathrm{~mL})$. The combined organic layers were dried $\left(\mathrm{Na}_{2} \mathrm{SO}_{4}\right)$ and concentrated under reduced pressure. The crude product was purified by silica gel column chromatography using $\mathrm{CH}_{2} \mathrm{Cl}_{2}$ /petroleum ether (9:1) as eluent to give 12 (191 $\mathrm{mg}, 96 \%$ ) as colorless oil. ${ }^{1} \mathrm{H}$ NMR (400 MHz, $\left.\mathrm{CDCl}_{3}\right): \delta=4.32$ (s, 2H), 1.8 (br s, $\left.1 \mathrm{H}, O H\right) \mathrm{ppm} ;{ }^{13} \mathrm{C}\left\{{ }^{1} \mathrm{H}\right\}$ NMR (100 MHz, $\left.\mathrm{CDCl}_{3}\right): \delta=73.1,70.7,64.7,51.3,41.8 \mathrm{ppm}$.

\section{Direct Conversion of Compound 20 to 12:}

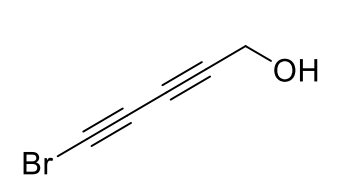

To a stirred solution of alkyne 20 (200 mg, $1.31 \mathrm{mmol})$ in acetone (4 mL), was added sequentially NBS (257 mg, $1.44 \mathrm{mmol}, 1.1$ equiv), silver nitrate (22.2 $\mathrm{mg}, 0.131 \mathrm{mmol}, 10 \mathrm{~mol} \%$ ) and $\mathrm{H}_{2} \mathrm{O}(0.1 \mathrm{~mL})$ at room temperature and the mixture stirred for $1 \mathrm{~h}$. After completion of the reaction (monitored by TLC), the mixture was cooled to $0{ }^{\circ} \mathrm{C}$ and quenched with cold water $(1 \mathrm{~mL})$. The reaction mixture was extracted with $\mathrm{Et}_{2} \mathrm{O}(3 \times 10 \mathrm{~mL})$. The combined organic layers were dried $\left(\mathrm{Na}_{2} \mathrm{SO}_{4}\right)$ and concentrated under reduced pressure. The crude product was purified by silica gel column chromatography using $\mathrm{CH}_{2} \mathrm{Cl}_{2}$ /petroleum ether (9:1) as eluent to give $\mathbf{1 2}$ (184 mg, 88\%) as colorless oil.

(4S,5S)-4-(tert-Butyldimethylsilyloxy)-5-(8-hydroxyocta-2,4,6-triyn-1-yl) $2(3 H)$-one (22)

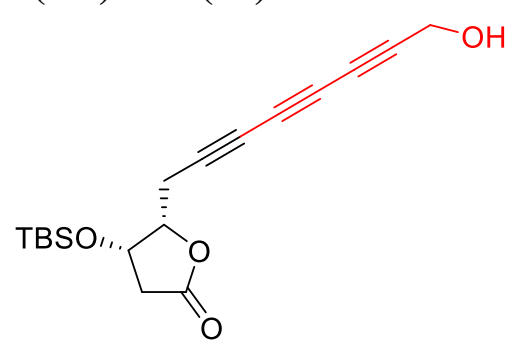

To the flask containing $\mathrm{CuCl}(2.0 \mathrm{mg}, 0.0196 \mathrm{mmol}, 10 \mathrm{~mol} \%)$ was added a $30 \%$ aq. solution of $n-\mathrm{BuNH}_{2}(1.5 \mathrm{~mL})$ at room temperature under nitrogen to form a blue color solution. A few crystals of hydroxylamine hydrochloride were added to discharge the blue color. To this solution, was added alkyne 13 (50 mg,

$0.196 \mathrm{mmol}, 1.0$ equiv) in $\mathrm{CH}_{2} \mathrm{Cl}_{2}(2 \mathrm{~mL})$ at $0{ }^{\circ} \mathrm{C}$ to result in the formation of a yellow acetylide suspension that was immediately cooled with an ice-water mixture. To this reaction mixture, bromodiyne 12 (47 mg, $0.294 \mathrm{mmol}, 1.5$ equiv) was added at once. More crystals of hydroxylamine hydrochloride were added throughout the reaction as necessary to prevent the solution from turning blue or green. The reaction mixture was stirred at $0{ }^{\circ} \mathrm{C}$ for $1 \mathrm{~h}$ and then extracted with $\mathrm{CH}_{2} \mathrm{Cl}_{2}(4 \times 20 \mathrm{~mL})$. The combined organic layers were dried $\left(\mathrm{Na}_{2} \mathrm{SO}_{4}\right)$ and concentrated. The crude product was purified by silica gel column chromatography using petroleum ether/EtOAc (4:1) as eluent to afford the corresponding triacetylene $22(51 \mathrm{mg}, 78 \%)$ as pale yellow oil. $[\alpha]_{\mathrm{D}}{ }^{25}=+8.7\left(c=1.0, \mathrm{CHCl}_{3}\right)$; IR $\left(\mathrm{CHCl}_{3}\right): v_{\max }=3438,3020,2929,2858$, 2224, 1784, 1734, 1466, 1373, 1351, 1154, 1065, 1025, 924, $668 \mathrm{~cm}^{-1} ;{ }^{1} \mathrm{H}\left(400 \mathrm{MHz}, \mathrm{CDCl}_{3}\right): \delta$ $=4.54(\mathrm{dt}, J=8.6,4.8 \mathrm{~Hz}, 1 \mathrm{H}), 4.51-4.48(\mathrm{~m}, 1 \mathrm{H}), 4.35(\mathrm{~s}, 2 \mathrm{H}), 2.83(\mathrm{~d}, J=7.0 \mathrm{~Hz}, 2 \mathrm{H}), 2.75$ 
$(\mathrm{dd}, J=17.4,5.1 \mathrm{~Hz}, 1 \mathrm{H}), 2.48(\mathrm{dd}, J=17.4,1.0 \mathrm{~Hz}, 1 \mathrm{H}), 0.89(\mathrm{~s}, 9 \mathrm{H}), 0.13(\mathrm{~s}, 3 \mathrm{H}), 0.10(\mathrm{~s}, 3 \mathrm{H})$ ppm; ${ }^{13} \mathrm{C}\left\{{ }^{1} \mathrm{H}\right\}$ NMR $\left(125 \mathrm{MHz}, \mathrm{CHCl}_{3}\right): \delta=174.4,81.6,75.5,75.1,70.7,68.6,67.5,63.5,59.9$, 51.5, 39.4, 25.6, 19.8, 18.0, -4.6, -5.2 ppm; HRMS (ESI-TOF) $m / z:\left[\mathrm{M}+\mathrm{Na}^{+}\right.$Calcd for $\mathrm{C}_{18} \mathrm{H}_{24} \mathrm{O}_{4} \mathrm{SiNa} 355.1333$; Found 355.1336.

(4S,5S)-4,8-Dihydroxy-3,4-dihydrovernoniyne (1b)

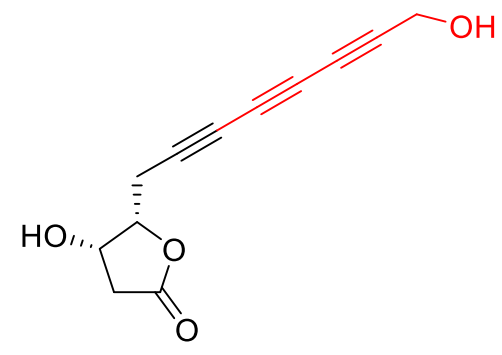

To a stirred solution of $22(30 \mathrm{mg}, 0.090 \mathrm{mmol})$ in $\mathrm{MeOH}(2 \mathrm{~mL})$ was added $2 \mathrm{M} \mathrm{HCl}(0.5 \mathrm{~mL})$ at $0{ }^{\circ} \mathrm{C}$. The reaction mixture was then stirred at $0{ }^{\circ} \mathrm{C}$ for $3 \mathrm{~h}$. The solution was diluted with EtOAc $(5 \mathrm{~mL})$ and the organic layer was washed with $\mathrm{NaHCO}_{3}$, brine, dried $\left(\mathrm{Na}_{2} \mathrm{SO}_{4}\right)$ and concentrated. The crude product was purified by silica gel column chromatography using petroleum ether/EtOAc (1:1) to afford $\mathbf{1 b}$ (17.3 $\mathrm{mg}$, $88 \%)$ as a pale yellow solid, Mp. $106-109{ }^{\circ} \mathrm{C} ;[\alpha]_{\mathrm{D}}{ }^{25}=-17.6(c=0.83$, EtOH$)$, lit. $^{4}$ M.p. $108-110$ ${ }^{\circ} \mathrm{C} ;[\alpha]_{\mathrm{D}}{ }^{25}=-61.5(c=0.94, \mathrm{EtOH}) ; \mathrm{IR}\left(\mathrm{CHCl}_{3}\right): v_{\max }=3416,3024,2928,2858,2220,1774,1453$, 1407, 1350, 1152, 1104, 1021, 924, 901, 809, $667 \mathrm{~cm}^{-1} ;{ }^{1} \mathrm{HNMR}\left(500 \mathrm{MHz}\right.$, Acetone- $\left.d_{6}\right): \delta=4.83$ $(\mathrm{d}, J=4.4 \mathrm{~Hz}, 1 \mathrm{H}), 4.64(\mathrm{ddd}, J=7.6,6.0,3.8 \mathrm{~Hz}, 1 \mathrm{H}), 4.61-4.59(\mathrm{~m}, 1 \mathrm{H}), 4.52(\mathrm{t}, J=6.1 \mathrm{~Hz}$, $1 \mathrm{H}), 4.31(\mathrm{~d}, J=6.0 \mathrm{~Hz}, 2 \mathrm{H}), 2.93(\mathrm{dd}, J=17.4,5.3 \mathrm{~Hz}, 1 \mathrm{H}), 2.89-2.81(\mathrm{~m}, 2 \mathrm{H}), 2.42(\mathrm{dd}, J=$ $17.4,1.1 \mathrm{~Hz}, 1 \mathrm{H}) \mathrm{ppm} ;{ }^{13} \mathrm{C}\left\{{ }^{1} \mathrm{H}\right\} \mathrm{NMR}\left(100 \mathrm{MHz}\right.$, Acetone- $\left.d_{6}\right): \delta=175.4,82.2,78.9,78.0,69.2$, 68.7, 66.8, 63.5, 60.5, 51.0, 39.8, 20.5 ppm; HRMS (ESI-TOF) $m / z:[\mathrm{M}+\mathrm{H}]^{+}$Calcd for $\mathrm{C}_{12} \mathrm{H}_{11} \mathrm{O}_{4}$ 219.0652; Found 219.0659.

\section{$(4 S, 5 R)-4-H y d r o x y-5-v i n y l d i h y d r o f u r a n-2(3 H)$-one $(16)^{5}$}

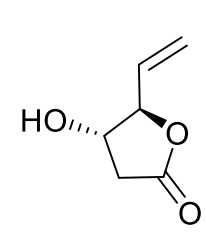

To a stirred solution of lactone $14(0.3 \mathrm{~g}, 2.34 \mathrm{mmol})$ in dry THF (4 mL) were added sequentially at room temperature, triphenylphosphine $(0.245 \mathrm{~g}, 0.936 \mathrm{mmol}, 40 \mathrm{~mol}$ $\%)$ and $\mathrm{Pd}(\mathrm{OAc})_{2}(53 \mathrm{mg}, 0.234 \mathrm{mmol}, 10 \mathrm{~mol} \%)$. The mixture was stirred at room temperature for $6 \mathrm{~h}$ and then the volatiles were evaporated under reduced pressure.

Analysis of the crude product indicated 14:16 = 10:90 mixture (by ${ }^{1} \mathrm{H}$ NMR). The residue was purified by silica gel column chromatography using petroleum ether/EtOAc (7:3) as eluent to afford $16(0.232 \mathrm{~g}, 88 \%$, based on recovered 14) as a colorless oil. Further elution gave the starting material $14(36 \mathrm{mg}, 12 \%)$ as colorless oil. Data for 16: $[\alpha]_{\mathrm{D}}^{25}=+24.5\left(c=1.0, \mathrm{CHCl}_{3}\right)$; IR $\left(\mathrm{CHCl}_{3}\right)$ : $v_{\max }=3445,2932,2852,1771,1646,1416,1360,1265,1179,1101,1061,990,936,879 \mathrm{~cm}^{-1} ;{ }^{1} \mathrm{H}$ NMR (400 MHz, $\left.\mathrm{CDCl}_{3}\right): 5.86(\mathrm{ddd}, J=17.1,10.7,5.3 \mathrm{~Hz}, 1 \mathrm{H}), 5.42$ (d, $\left.J=17.2 \mathrm{~Hz}, 1 \mathrm{H}\right), 5.31$ $(\mathrm{d}, J=10.7 \mathrm{~Hz}, 1 \mathrm{H}), 4.85(\mathrm{~s}, 1 \mathrm{H}), 4.38-4.36(\mathrm{~m}, 1 \mathrm{H}), 2.79$ (dd, $J=17.8,6.2 \mathrm{~Hz}, 1 \mathrm{H}), 2.50$ (dd, $J$ 
$=17.8,3.3 \mathrm{~Hz}, 1 \mathrm{H}), 2.04(\mathrm{br} \mathrm{s}, 1 \mathrm{H}, \mathrm{OH}) \mathrm{ppm} ;{ }^{13} \mathrm{C}\left\{{ }^{1} \mathrm{H}\right\} \mathrm{NMR}\left(125 \mathrm{MHz}, \mathrm{CDCl}_{3}\right): \delta=175.1,132.7$, 118.3, 87.3 , 71.8, 36.7 ppm; HRMS (ESI-TOF) $m / z:[\mathrm{M}+\mathrm{H}]^{+}$Calcd for $\mathrm{C}_{6} \mathrm{H}_{9} \mathrm{O}_{3}$ 129.0552; Found 129.0555 .

(4S,5R)-4-(tert-Butyldimethylsilyloxy)-5-vinyldihydrofuran-2(3H)-one (23)

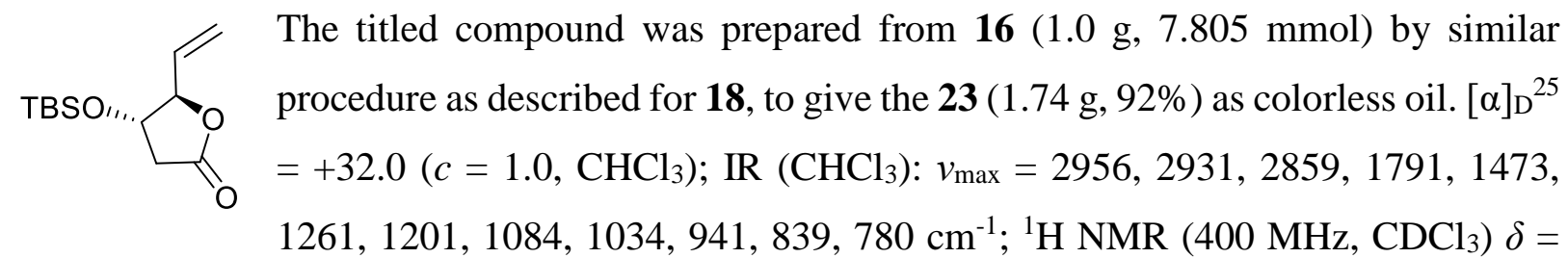
$5.81(\mathrm{ddd}, J=17.0,10.6,5.7 \mathrm{~Hz}, 1 \mathrm{H}), 5.42(\mathrm{dt}, J=17.4,1.1 \mathrm{~Hz}, 1 \mathrm{H}), 5.30(\mathrm{dt}, J=10.5,1.0 \mathrm{~Hz}$, $1 \mathrm{H}), 4.70(\mathrm{dd}, J=7.2,4.2 \mathrm{~Hz}, 1 \mathrm{H}), 4.26-4.22(\mathrm{~m}, 1 \mathrm{H}), 2.72(\mathrm{dd}, J=17.4,6.4 \mathrm{~Hz}, 1 \mathrm{H}), 2.41(\mathrm{dd}$, $J=17.4,4.2 \mathrm{~Hz}, 1 \mathrm{H}), 0.87$ (s, 9H), 0.07 (s, 3H), 0.06 (s, 3H) ppm; ${ }^{13} \mathrm{C}\left\{{ }^{1} \mathrm{H}\right\} \mathrm{NMR}(100 \mathrm{MHz}$, $\left.\mathrm{CDCl}_{3}\right): \delta=174.7,132.9,118.2,87.6,72.6,37.4,25.6,17.9,-4.8,-4.9$ ppm; HRMS (ESI-TOF) $m / z:[\mathrm{M}+\mathrm{H}]^{+}$Calcd for $\mathrm{C}_{12} \mathrm{H}_{23} \mathrm{O}_{3} \mathrm{Si}$ 243.1415; Found 243.1418.

\section{(4S,5R)-4-(tert-Butyldimethylsilyloxy)-5-(prop-2-yn-1-yl)dihydrofuran-2(3H)-one (24)}

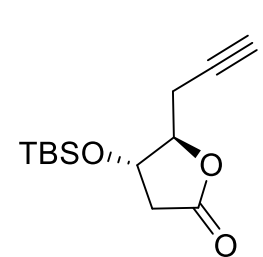

The titled compound was prepared from $23(0.150 \mathrm{~g}, 0.619 \mathrm{mmol})$ by a similar procedure as described for $\mathbf{1 3}$, to give the corresponding alkyne $\mathbf{2 4}$ (85 $\mathrm{mg}, 54 \%$ ) as colorless oil. $[\alpha]_{\mathrm{D}}^{25}=+52.3\left(c=1.0, \mathrm{CHCl}_{3}\right)$; IR $\left(\mathrm{CHCl}_{3}\right)$ : $v_{\max }=3308,3019$, 2955, 2931, 2858, 2400, 1786, 1523, 1471, 1420, 1296, 1251, 1153, 1115, 1082, 1037, 977, 926, 839, $669 \mathrm{~cm}^{-1} ;{ }^{1} \mathrm{H}$ NMR (500 MHz, $\left.\mathrm{CDCl}_{3}\right): \delta=4.46(\mathrm{dt}, J=6.6,3.3 \mathrm{~Hz}, 1 \mathrm{H})$, $4.41-4.37(\mathrm{~m}, 1 \mathrm{H}), 2.86(\mathrm{dd}, J=17.8,6.7 \mathrm{~Hz}, 1 \mathrm{H}), 2.64-2.52(\mathrm{~m}, 2 \mathrm{H}), 2.45(\mathrm{dd}, J=17.8,3.6 \mathrm{~Hz}$, $1 \mathrm{H}), 2.07(\mathrm{t}, J=2.6 \mathrm{~Hz}, 1 \mathrm{H}), 0.88(\mathrm{~s}, 9 \mathrm{H}), 0.10(\mathrm{~s}, 3 \mathrm{H}), 0.09$ (s, 3H) ppm; ${ }^{13} \mathrm{C}$ NMR $(125 \mathrm{MHz}$, $\mathrm{CDCl}_{3}$ ): $\delta=174.6,84.7,77.8,71.9,70.9,38.1,25.6,22.4,17.8,-4.8,-4.9$ ppm; HRMS (ESITOF) $m / z:[\mathrm{M}+\mathrm{Na}]^{+}$Calcd for $\mathrm{C}_{13} \mathrm{H}_{22} \mathrm{O}_{3} \mathrm{SiNa} 277.1230$; Found 277.1234.

(4S,5R)-4-(tert-Butyldimethylsilyloxy)-5-(8-hydroxyocta-2,4,6-triyn-1-yl) dihydrofuran2(3H)-one (25)

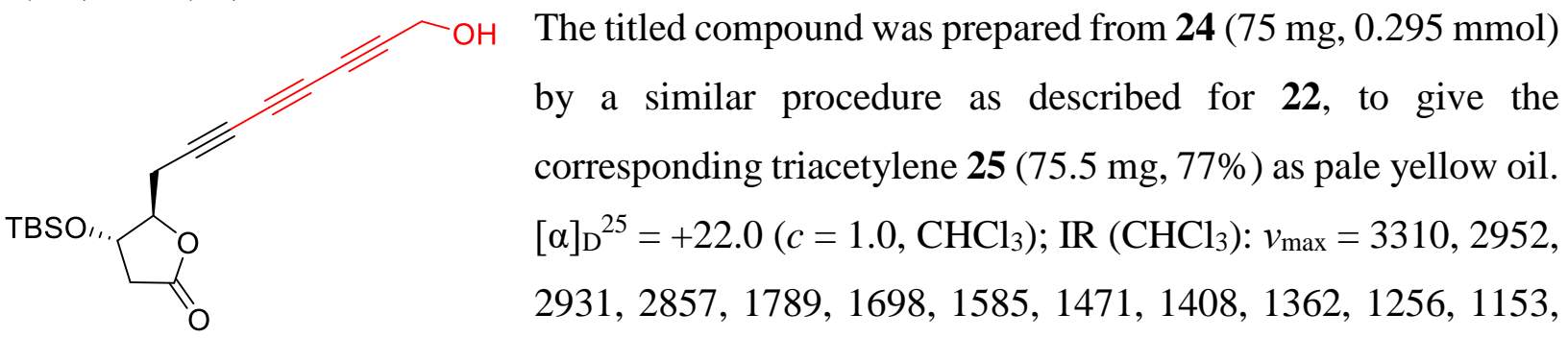

1103, 1082, 1039, 978, 840, $668 \mathrm{~cm}^{-1} ;{ }^{1} \mathrm{H}$ NMR (500 MHz, $\left.\mathrm{CDCl}_{3}\right): \delta=4.42-4.36(\mathrm{~m}, 2 \mathrm{H}), 4.35$ (s, 2H), $2.84(\mathrm{dd}, J=17.8,6.6 \mathrm{~Hz}, 1 \mathrm{H}), 2.78-2.63(\mathrm{~m}, 2 \mathrm{H}), 2.48(\mathrm{dd}, J=17.8,4.0 \mathrm{~Hz}, 1 \mathrm{H}), 1.73$ 
$(\mathrm{s}, 1 \mathrm{H}), 0.89(\mathrm{~s}, 9 \mathrm{H}), 0.12(\mathrm{~s}, 3 \mathrm{H}), 0.10(\mathrm{~s}, 3 \mathrm{H}) \mathrm{ppm} ;{ }^{13} \mathrm{C}\left\{{ }^{1} \mathrm{H}\right\} \mathrm{NMR}\left(125 \mathrm{MHz}, \mathrm{CDCl}_{3}\right): \delta=174.0$, 84.1, 75.8, 73.8, 71.1, 70.5, 68.5, 63.1, 60.5, 51.5, 38.0, 25.6, 23.5, 17.8, -4.8, -4.9 ppm; HRMS (ESI-TOF) $m / z:[\mathrm{M}+\mathrm{Na}]^{+}$Calcd for $\mathrm{C}_{18} \mathrm{H}_{24} \mathrm{O}_{4} \mathrm{SiNa} 355.1333$; Found 355.1338.

\section{(4S,5R)-4,8-Dihydroxy-3,4-dihydrovernoniyne (1a)}

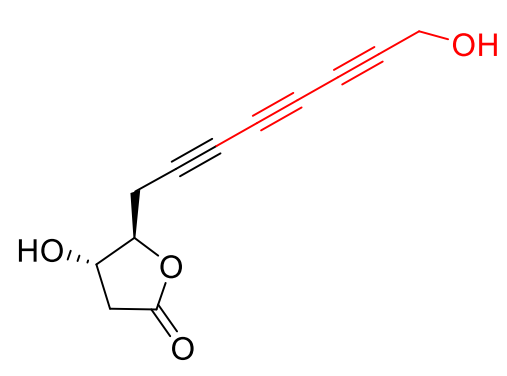

The titled compound was prepared from 25 (45 mg, $0.135 \mathrm{mmol})$ by a similar procedure as described for $\mathbf{1 b}$, to give $\mathbf{1 a}(26.5 \mathrm{mg}$, $90 \%$ ) as a pale yellow solid, M.p. $=106-109^{\circ} \mathrm{C},[\alpha]_{\mathrm{D}}{ }^{25}=+12.0$ $(c=0.75$, EtOH $)$, lit. ${ }^{4}$ M.p. $=108-110{ }^{\circ} \mathrm{C} ;[\alpha]_{\mathrm{D}}^{25}=+4.61(c=$ 0.76, EtOH); IR $\left(\mathrm{CHCl}_{3}\right): v_{\max }=3308,3020,2960,2854,2765$, 2400, 2257, 1786, 1739, 1613, 1463, 1427, 1412, 1363, 1339, 1288, 1259, 1146, 1114, 1081, 1037, 977, 891, 840, 668, $627 \mathrm{~cm}^{-1} ;{ }^{1} \mathrm{H}$ NMR (500 MHz, Acetone$\left.d_{6}\right): \delta=4.90(\mathrm{~d}, J=4.0 \mathrm{~Hz}, 1 \mathrm{H}), 4.53(\mathrm{t}, J=6.2 \mathrm{~Hz}, 1 \mathrm{H}), 4.47(\mathrm{dd}, J=6.5,3.9 \mathrm{~Hz}, 2 \mathrm{H}), 4.31(\mathrm{~d}, J$ $=6.2 \mathrm{~Hz}, 2 \mathrm{H}), 2.95(\mathrm{dd}, J=17.9,6.4 \mathrm{~Hz}, 1 \mathrm{H}), 2.88-2.80(\mathrm{~m}, 2 \mathrm{H}), 2.42(\mathrm{dd}, J=17.9,3.3 \mathrm{~Hz}, 1 \mathrm{H})$ ppm; ${ }^{13} \mathrm{C}\left\{{ }^{1} \mathrm{H}\right\}$ NMR $\left(125 \mathrm{MHz}\right.$, Acetone- $\left.d_{6}\right): \delta=175.2,85.1,79.1,77.0,71.1,69.1,67.6,63.2$, 60.8, 60.0, 37.7, 24.0 ppm; HRMS (ESI-TOF) $m / z:[\mathrm{M}+\mathrm{H}]^{+}$Calcd for $\mathrm{C}_{12} \mathrm{H}_{11} \mathrm{O}_{4} 219.0652$; Found 219.0657 .

\section{$(4 R, 5 R)-4-H y d r o x y-5-v i n y l-d i h y d r o f u r a n-2(3 H)-o n e ~(e n t-14)^{6}$}

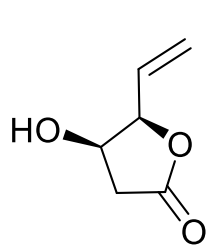

The titled compound was prepared from D-glucono- $\delta$-lactone 17 (4.0 g, 22.45 mmol) by a similar procedure as described for $\mathbf{1 4}$ to give the corresponding $\gamma$ hydroxylactone ent-14 $(1.47 \mathrm{~g}, 51 \%)$ as colorless oil. $[\alpha]_{\mathrm{D}}{ }^{25}=+44.5(c=1.0$, $\mathrm{CHCl}_{3}$ ). Other spectral data is same as its enantiomer 14.

(4R,5R)-4-(tert-Butyldimethylsilyloxy)-5-vinyldihydrofuran-2(3H)-one (ent-18)

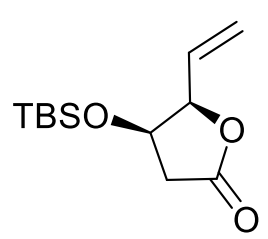

The titled compound was prepared from ent-14 (100 mg, $0.780 \mathrm{mmol})$ by a similar procedure as described for 18, to give ent-18 (176 $\mathrm{mg}, 93 \%)$ as colorless oil, $[\alpha]_{\mathrm{D}}^{25}=+15.5\left(c=1.0, \mathrm{CHCl}_{3}\right)$. Other spectral data is same as its enantiomer 18.

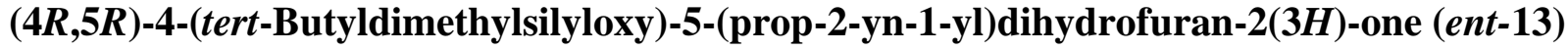

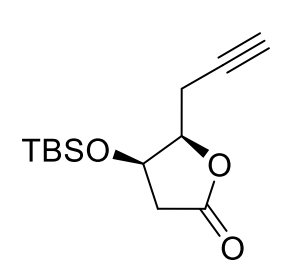

The titled compound was prepared from ent-18 (100 $\mathrm{mg}, 0.412 \mathrm{mmol})$ by a similar procedure as described for 13, to give the corresponding alkyne ent-13 $(58 \mathrm{mg}, 55 \%)$ as colorless oil, $[\alpha]_{\mathrm{D}}^{25}=-6.24\left(c=1.0, \mathrm{CHCl}_{3}\right)$. Other spectral data is same as its enantiomer 13. 


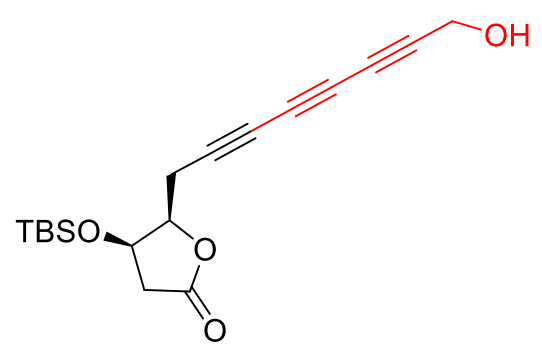

The titled compound was prepared from ent-13 $(60 \mathrm{mg}, 0.236$ $\mathrm{mmol}$ ) by a similar procedure as described for $\mathbf{2 2}$, to give the corresponding triacetylene ent-22 $(59.6 \mathrm{mg}, 76 \%)$ as pale yellow oil, $[\alpha]_{\mathrm{D}}{ }^{25}=-9.6\left(c=1.0, \mathrm{CHCl}_{3}\right)$. Other spectral data is same as its enantiomer 22.

(4R,5R)-4,8-Dihydroxy-3,4-dihydrovernoniyne (ent-1b)

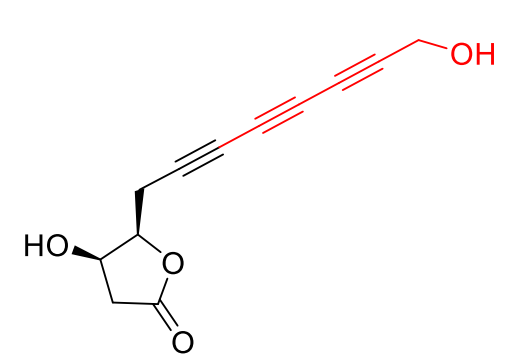

The titled compound was prepared from ent-22 $(60 \mathrm{mg}, 0.180$ $\mathrm{mmol}$ ) by a similar procedure as described for $\mathbf{1 b}$, to give the corresponding alcohol ent-1b (36 mg, 91\%) as pale yellow solid, M.p. $=105-107^{\circ} \mathrm{C},[\alpha]_{\mathrm{D}}{ }^{25}=+23.5(c=0.61$, EtOH $)$. Other spectral data is same as its enantiomer $\mathbf{1 b}$.

\section{(4R,5S)-4-Hydroxy-5-vinyl dihydrofuran-2(3H)-one (ent-16)}

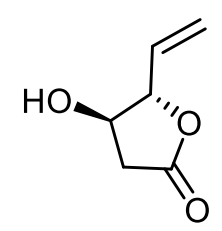

The titled compound was prepared from ent-14 $(200 \mathrm{mg}, 1.56 \mathrm{mmol})$ by a similar procedure as described for $\mathbf{1 6}$ to give the corresponding $\gamma$-hydroxylactone ent-16 as 89:11 mixture of diastereomers. Separation of this mixture gave ent-16 $(0.153 \mathrm{mg}$, $87 \%$, based on recovered ent -14$),[\alpha]_{\mathrm{D}}^{25}=-23.0\left(c=0.8, \mathrm{CHCl}_{3}\right)$. Other spectral data is same as its enantiomer $\mathbf{1 6 .}$

\section{(4R,5S)-4-(tert-Butyldimethylsilyloxy)-5-vinyldihydrofuran-2(3H)-one (ent-23)}<smiles>C=CC1OC(=O)CC1OC(C)(C)C</smiles>

The titled compound was prepared from ent-16 $(200 \mathrm{~g}, 1.56 \mathrm{mmol})$ by a similar procedure as described for 18, to give the TBS compound ent-23 (348 $\mathrm{mg}, 92 \%$ ) as colorless oil, $[\alpha]_{\mathrm{D}}{ }^{25}=-13.2\left(c=0.7, \mathrm{CHCl}_{3}\right)$. Other spectral data is same as its enantiomer 23.

\section{(4R,5S)-4-(tert-Butyldimethylsilyloxy)-5-(prop-2-yn-1-yl)dihydrofuran-2(3H)-one (ent-24)}

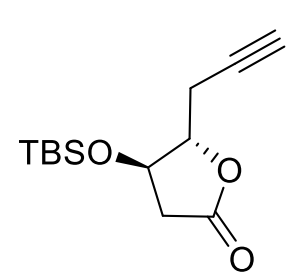

The titled compound was prepared from ent-23 $(180 \mathrm{mg}, 0.743 \mathrm{mmol})$ by a similar procedure as described for $\mathbf{1 3}$, to give the corresponding alkyne ent-24 $(103 \mathrm{mg}, 54 \%)$ as colorless oil, $[\alpha]_{\mathrm{D}}{ }^{25}=-50.3\left(c=0.8, \mathrm{CHCl}_{3}\right)$. Other spectral data is same as its enantiomer $\mathbf{2 4}$. 


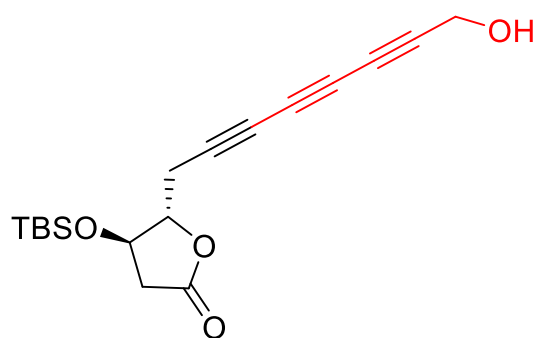

(4R,5S)-4,8-Dihydroxy-3,4-dihydrovernoniyne (ent-1a)

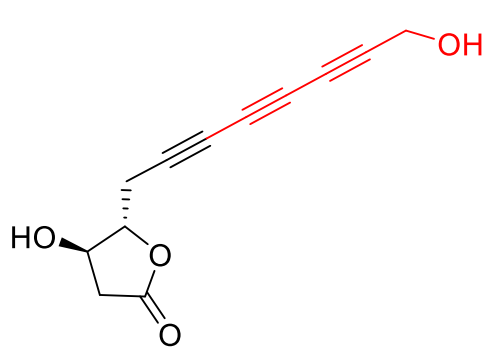
data is same as its enantiomer 1a.
The titled compound was prepared from ent-24 $(75 \mathrm{mg}, 0.295$ $\mathrm{mmol}$ ) by a similar procedure as described for $\mathbf{2 2}$, to give the corresponding triacetylene ent-25 (77.5 $\mathrm{mg}, 79 \%)$ as pale yellow oil, $[\alpha]_{\mathrm{D}}^{25}=-23.1\left(c=0.87, \mathrm{CHCl}_{3}\right)$. Other spectral data is same as its enantiomer $\mathbf{2 5}$.

The titled compound was prepared from ent-25 $(35 \mathrm{mg}, 0.105$ mmol) by a similar procedure as described for $\mathbf{1 b}$, to give the corresponding alcohol ent-1a (20.8 $\mathrm{mg}, 91 \%)$ as pale yellow solid, M.p. $=104-106{ }^{\circ} \mathrm{C} ;[\alpha]_{\mathrm{D}}{ }^{25}=-11.2(c=0.72$, EtOH$)$. Other spectral

\section{References:}

1. (a) Song, J.; Hollingsworth, R. I. Tetrahedron: Asymmetry 2001, 12, 387-391. (b) Chaudhari, D. A.; Kattanguru, P.; Fernandes, R. A. RSC Adv. 2015, 5, 42131-42134.

2. Evans, M. A.; Morken, J. P. Org. Lett. 2005, 7, 3371-3373.

3. Khomane, N. B; Meshram, H. M; Rode, H. B. Tetrahedron Lett. 2018, 59, 2157-2160.

4. Kanikarapu, S.; Marumudi, K; Kunwar, A. C.; Yadav, J. S.; Mohapatra, D. K. Org. Lett. 2017, 19, 4167-4170.

5. Fernandes, R. A.; Kattanguru, P.; Bethi, V. RSC Advances 2014, 4, 14507-14512.

6. (a) Fernandes, R. A.; Kattanguru, P. Asian J. Org. Chem. 2013, 2, 74-84. (b) Fernandes, R. A.; Kattanguru, P. J. Org. Chem. 2012, 77, 9357-9360. (c) Fernandes, R. A.; Kattanguru, P. Indian Patent 285997, 2017. (d) Fernandes, R. A.; Kattanguru, P. Indian Patent 292674, 2018. 
${ }^{1} \mathrm{H}$ NMR $\left(500 \mathrm{MHz}, \mathrm{CDCl}_{3}\right)$ and ${ }^{13} \mathrm{C} \mathrm{NMR}\left(125 \mathrm{MHz}, \mathrm{CDCl}_{3}\right)$ of compound 14
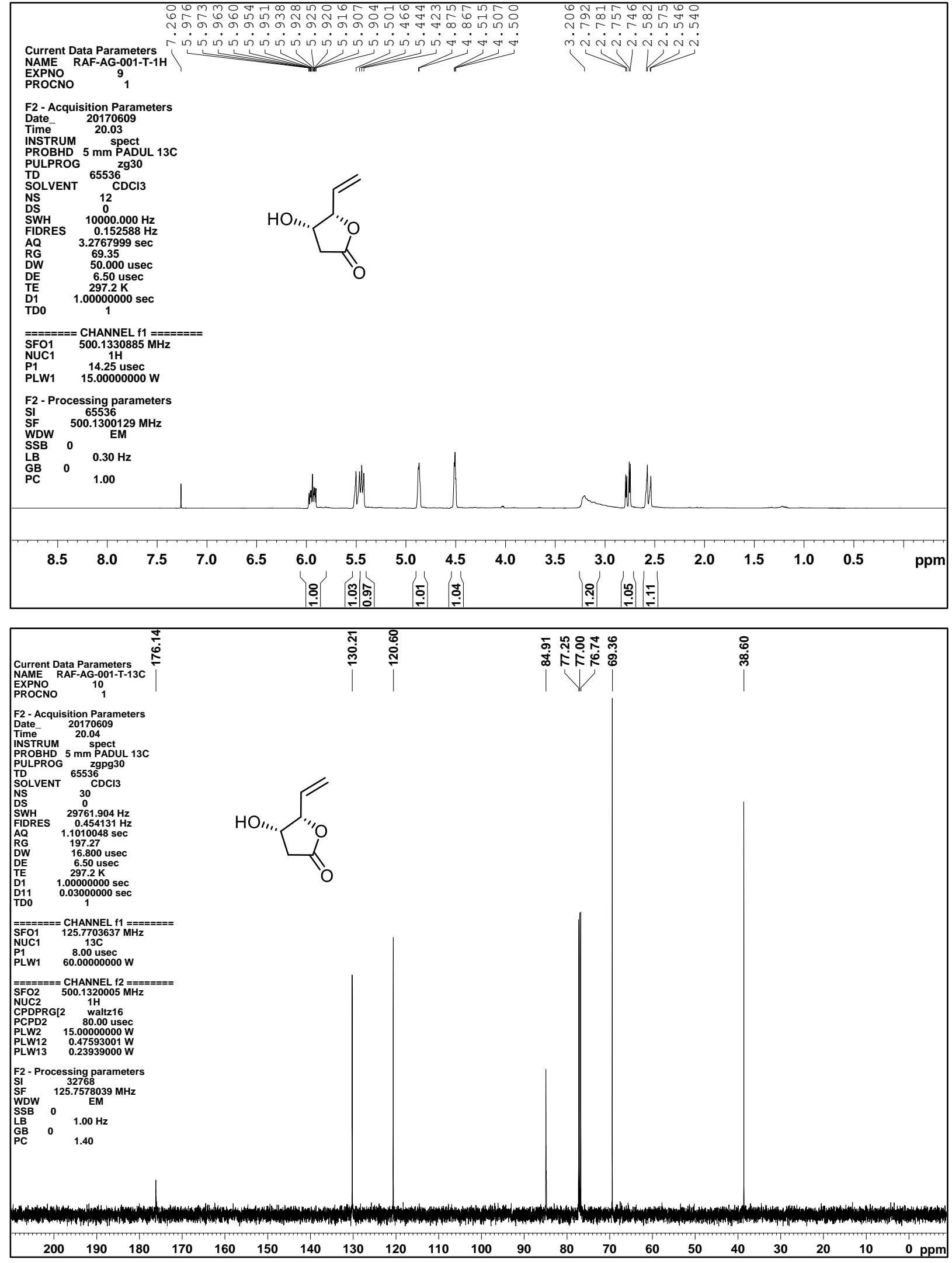
${ }^{1} \mathrm{H}$ NMR $\left(400 \mathrm{MHz}, \mathrm{CDCl}_{3}\right)$ and ${ }^{13} \mathrm{C}$ NMR $\left(100 \mathrm{MHz}, \mathrm{CDCl}_{3}\right)$ of compound 18
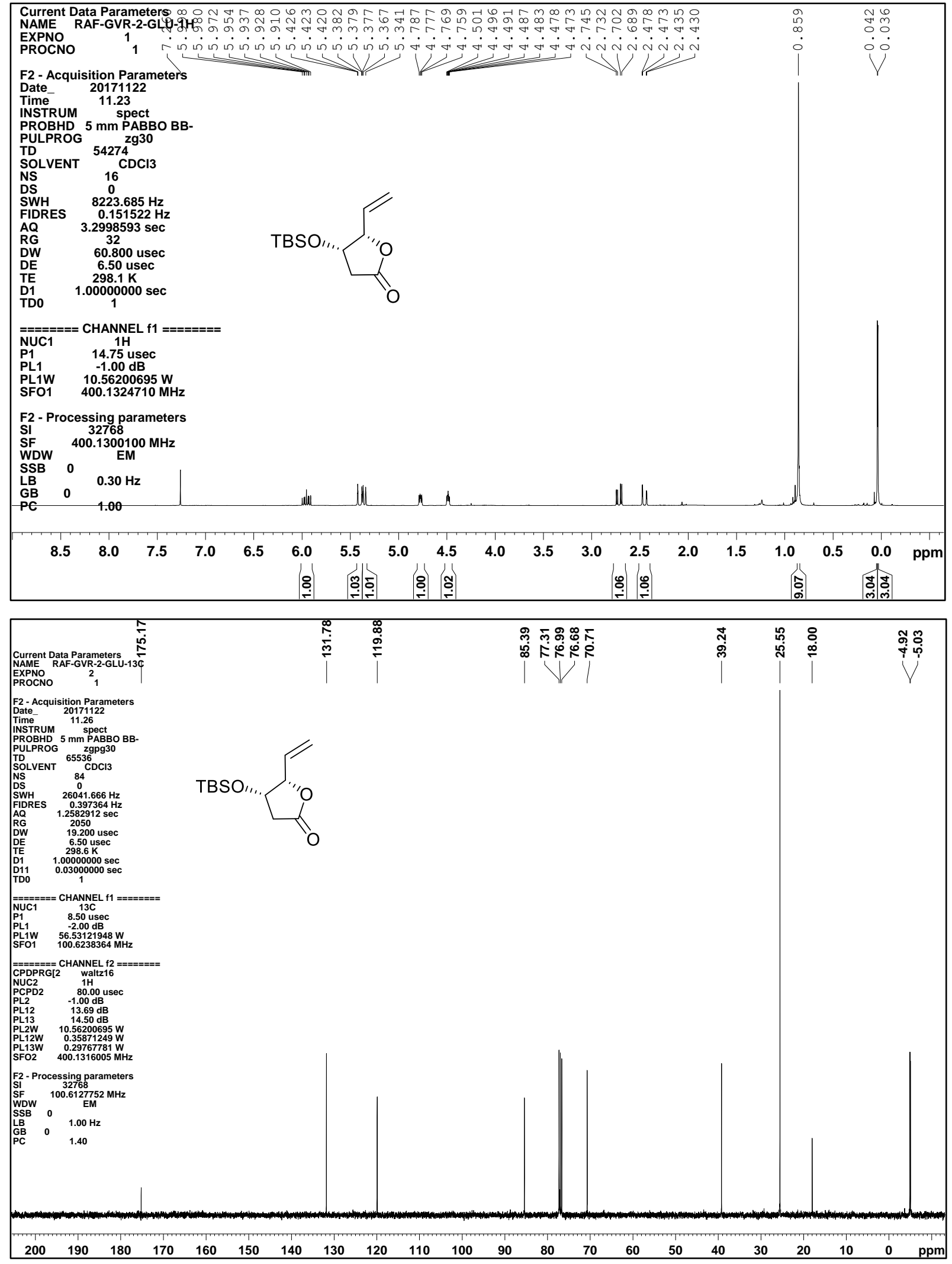
${ }^{1} \mathrm{H}$ NMR $\left(400 \mathrm{MHz}, \mathrm{CDCl}_{3}\right)$ and ${ }^{13} \mathrm{C}$ NMR $\left(100 \mathrm{MHz}, \mathrm{CDCl}_{3}\right)$ of compound 13
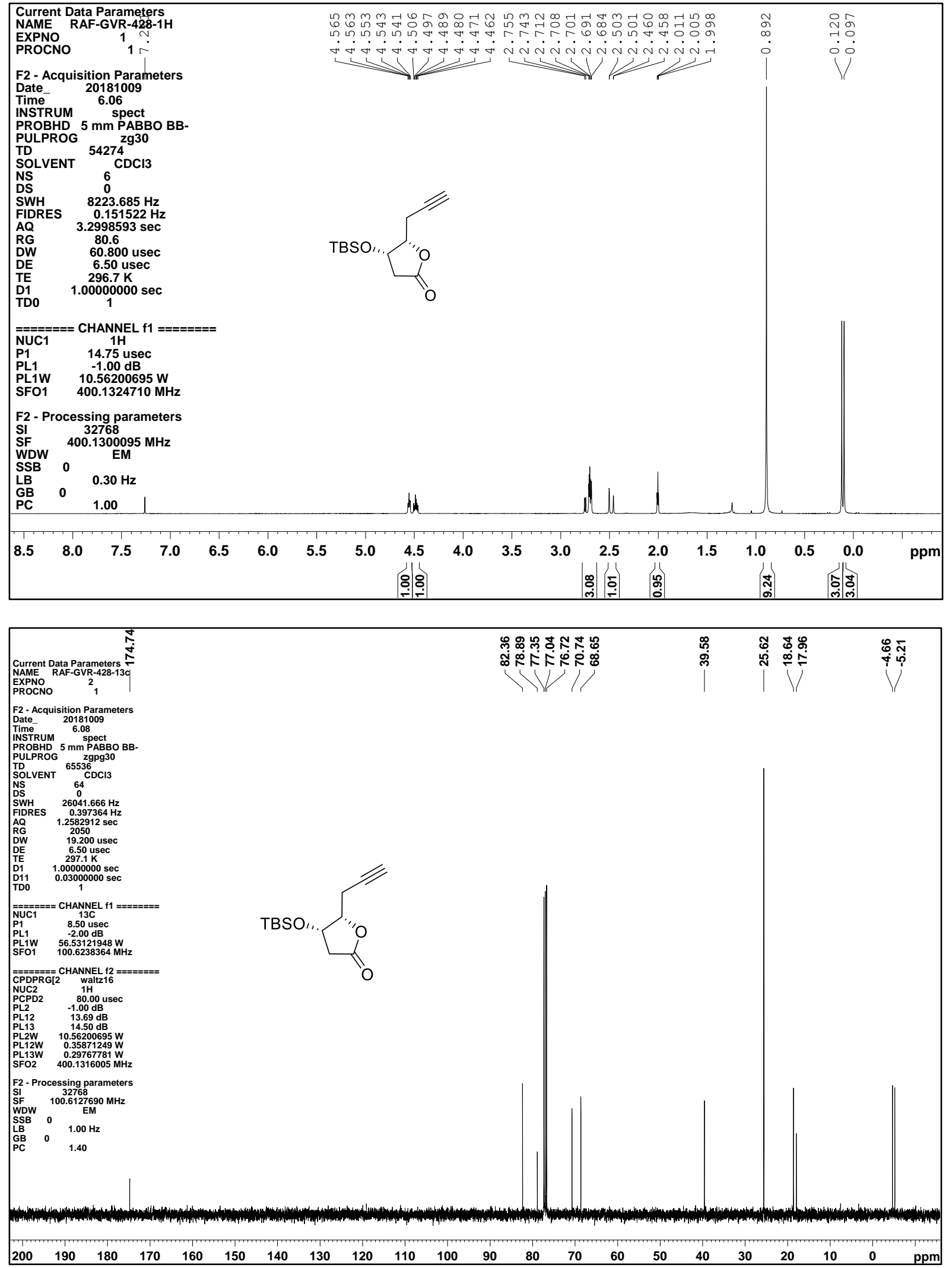
${ }^{1} \mathrm{H}$ NMR (400 MHz, $\mathrm{CDCl}_{3}$ ) and ${ }^{13} \mathrm{C}$ NMR $\left(100 \mathrm{MHz}, \mathrm{CDCl}_{3}\right)$ of compound 21
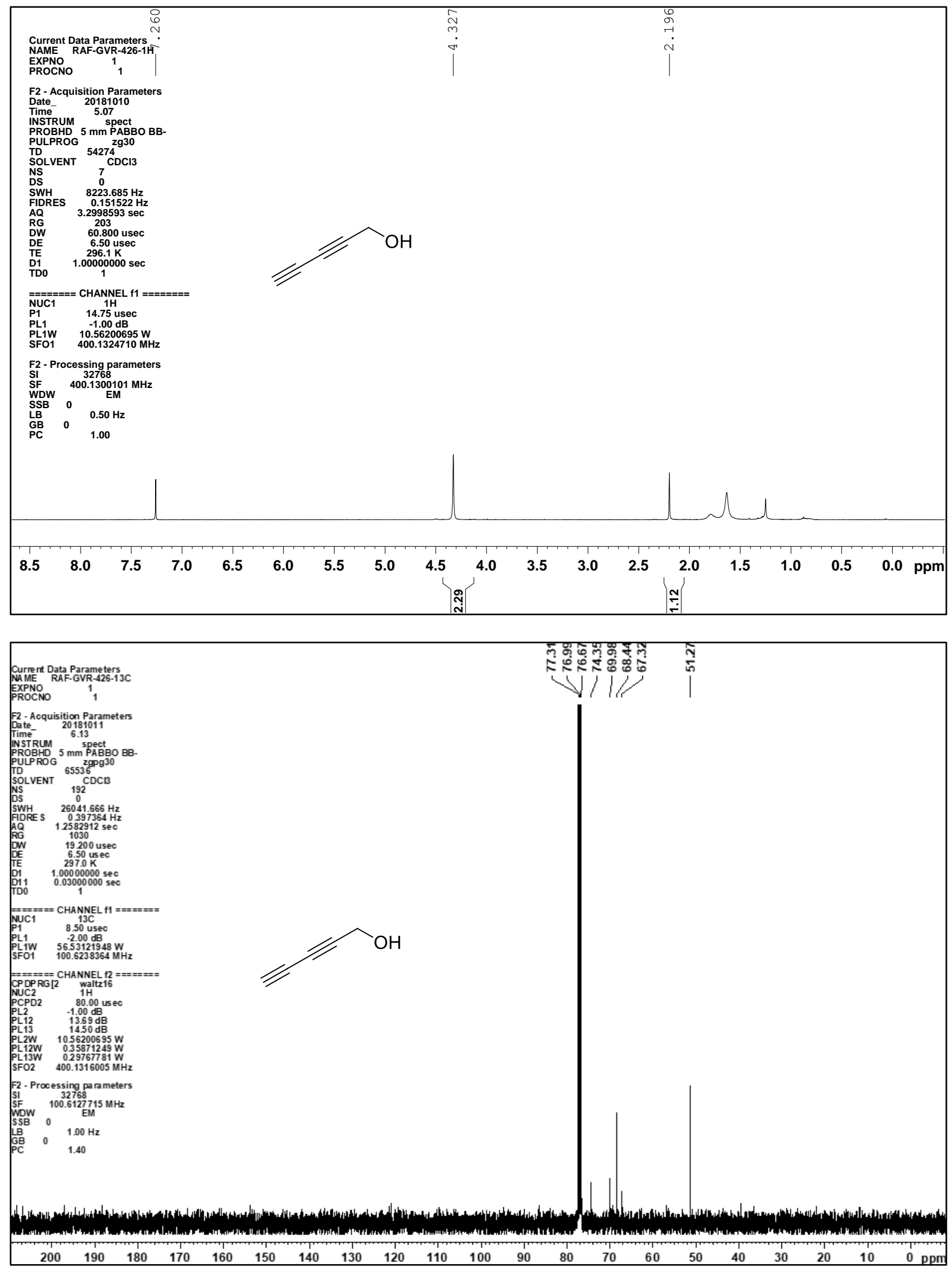
${ }^{1} \mathrm{H}$ NMR $\left(400 \mathrm{MHz}, \mathrm{CDCl}_{3}\right)$ and ${ }^{13} \mathrm{C}$ NMR $\left(100 \mathrm{MHz}, \mathrm{CDCl}_{3}\right)$ of compound 12
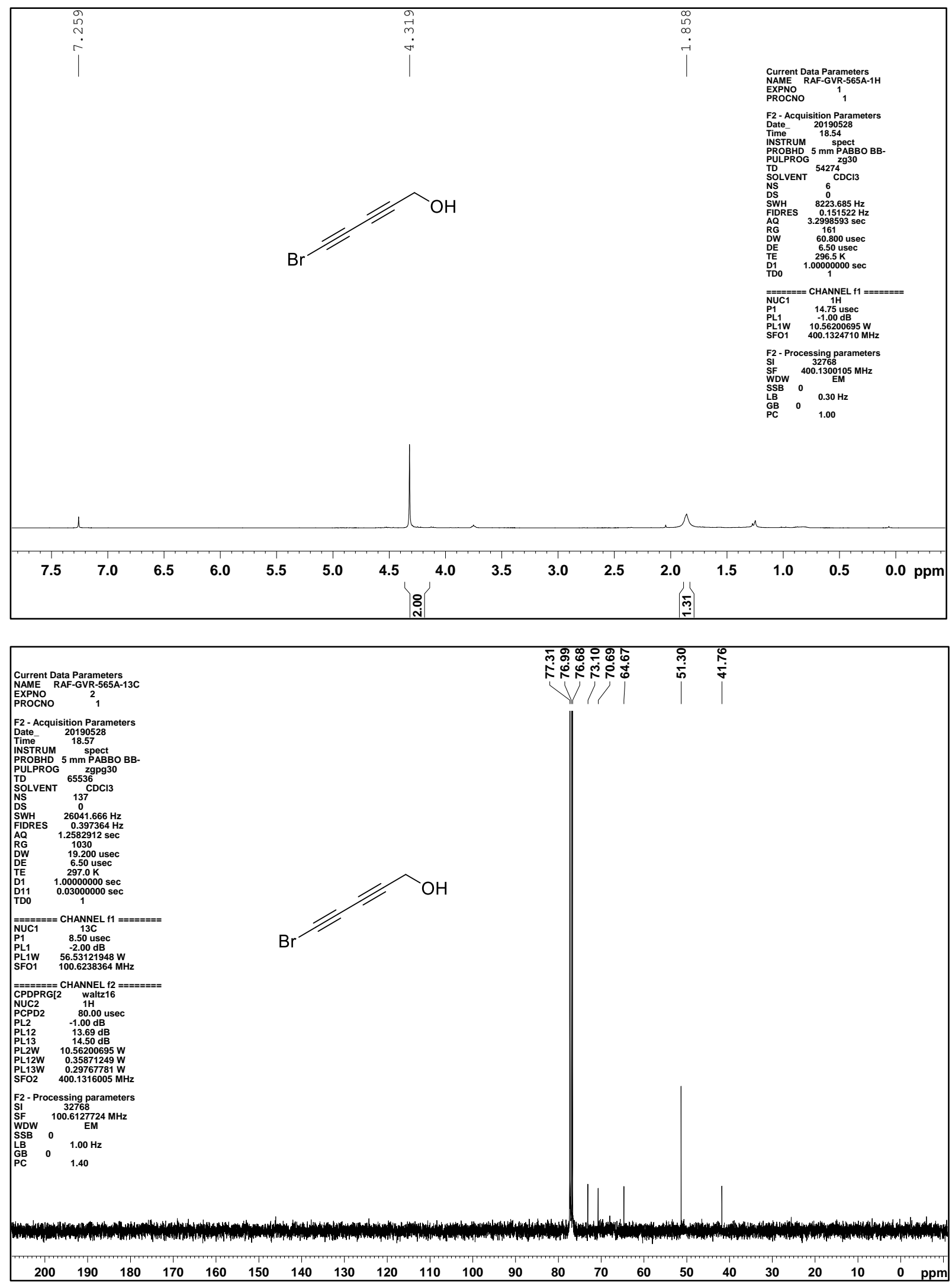
${ }^{1} \mathrm{H}$ NMR $\left(500 \mathrm{MHz}, \mathrm{CDCl}_{3}\right)$ and ${ }^{13} \mathrm{C}$ NMR $\left(125 \mathrm{MHz}, \mathrm{CDCl}_{3}\right)$ of compound 22
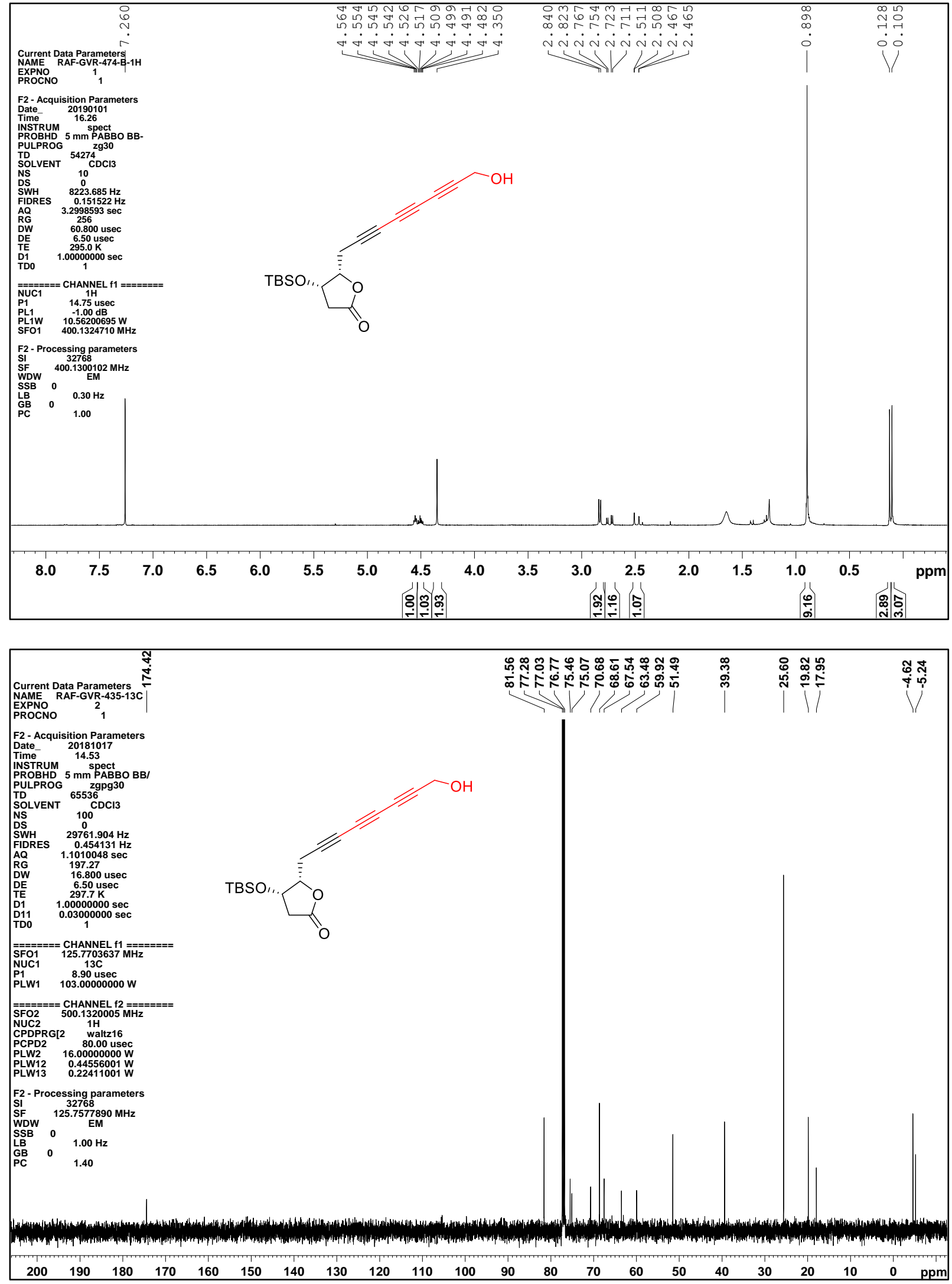
${ }^{1} \mathrm{H}$ NMR $\left(500 \mathrm{MHz}\right.$, acetone $d_{6}$ ) of compound $\mathbf{1 b}$ and known spectra
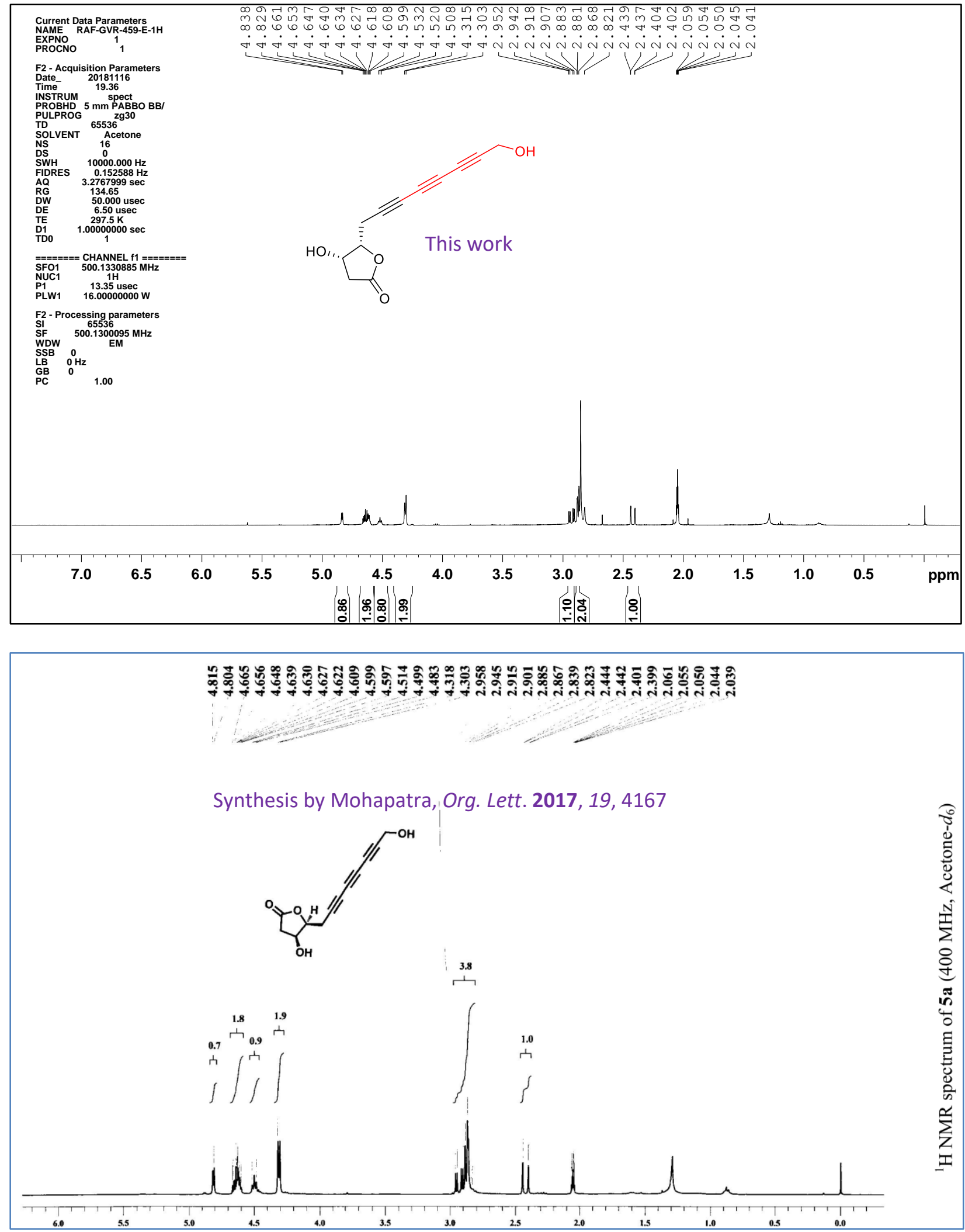
${ }^{13} \mathrm{C}$ NMR (125 MHz, acetone $d_{6}$ ) of compound $\mathbf{1 b}$ and known spectra
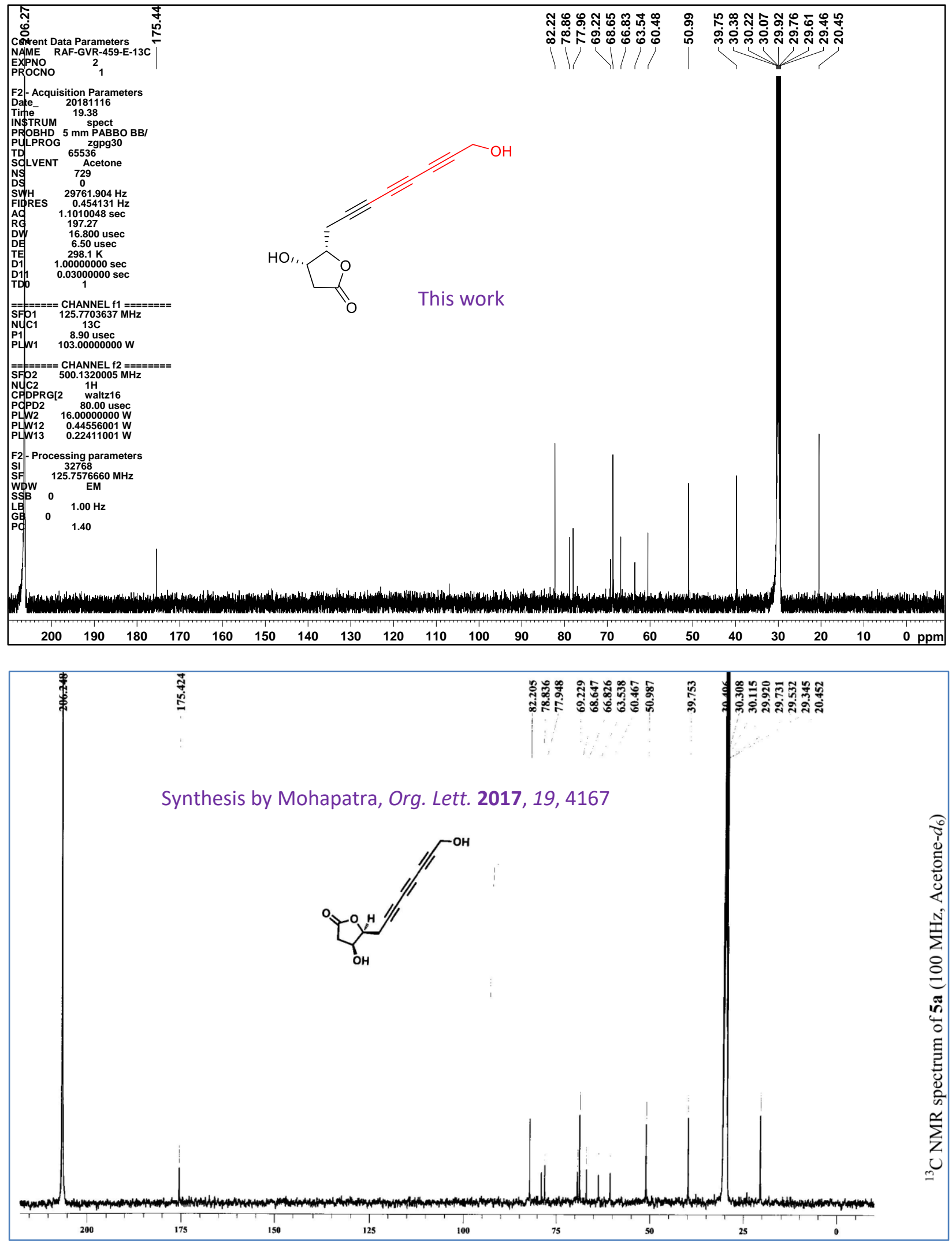
${ }^{1} \mathrm{H}$ NMR (400 MHz, $\mathrm{CDCl}_{3}$ ) and ${ }^{13} \mathrm{C}$ NMR $\left(125 \mathrm{MHz}, \mathrm{CDCl}_{3}\right.$ ) of compound 16
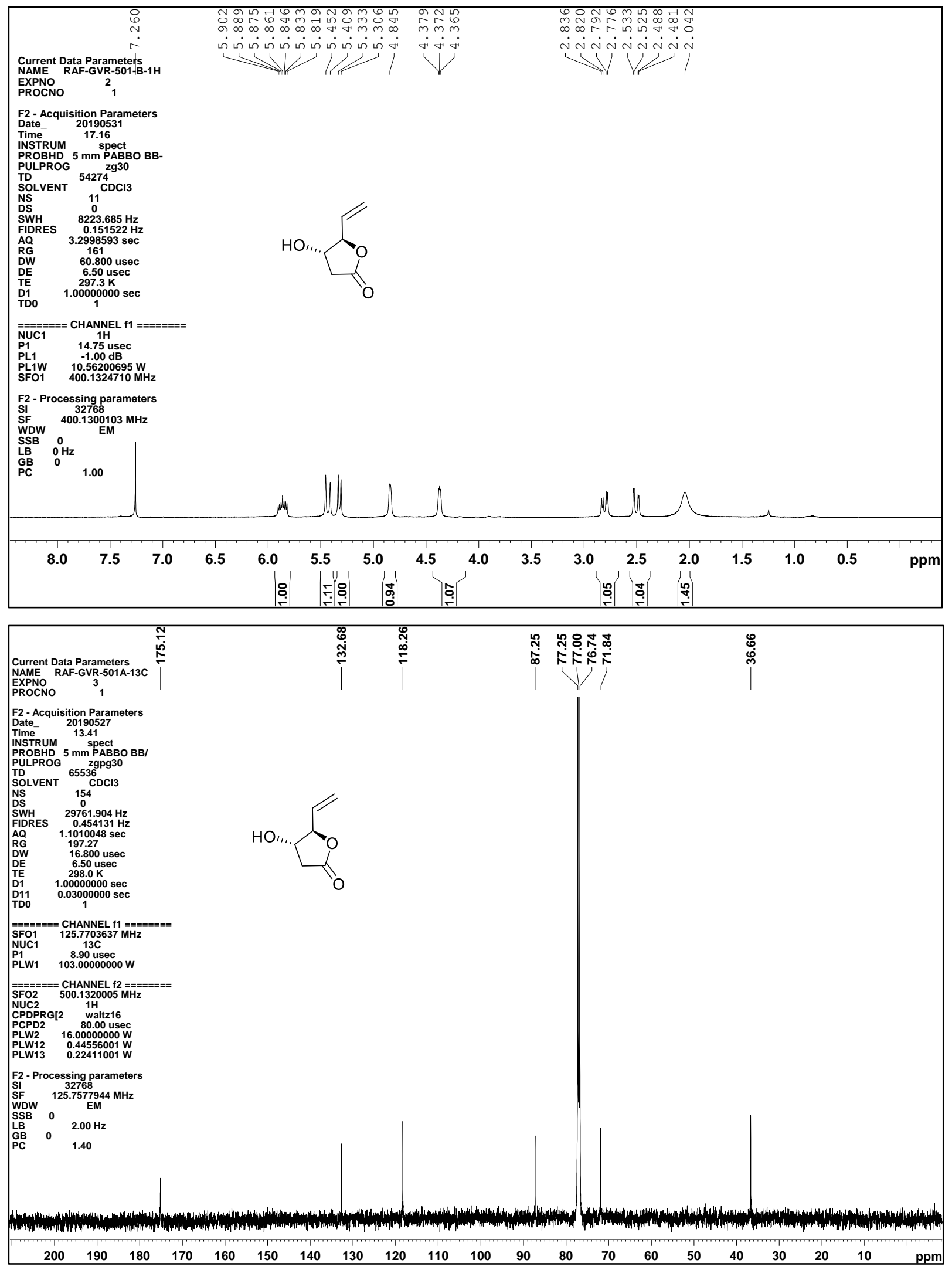
${ }^{1} \mathrm{H}$ NMR $\left(400 \mathrm{MHz}, \mathrm{CDCl}_{3}\right)$ and ${ }^{13} \mathrm{C}$ NMR $\left(100 \mathrm{MHz}, \mathrm{CDCl}_{3}\right)$ of compound 23
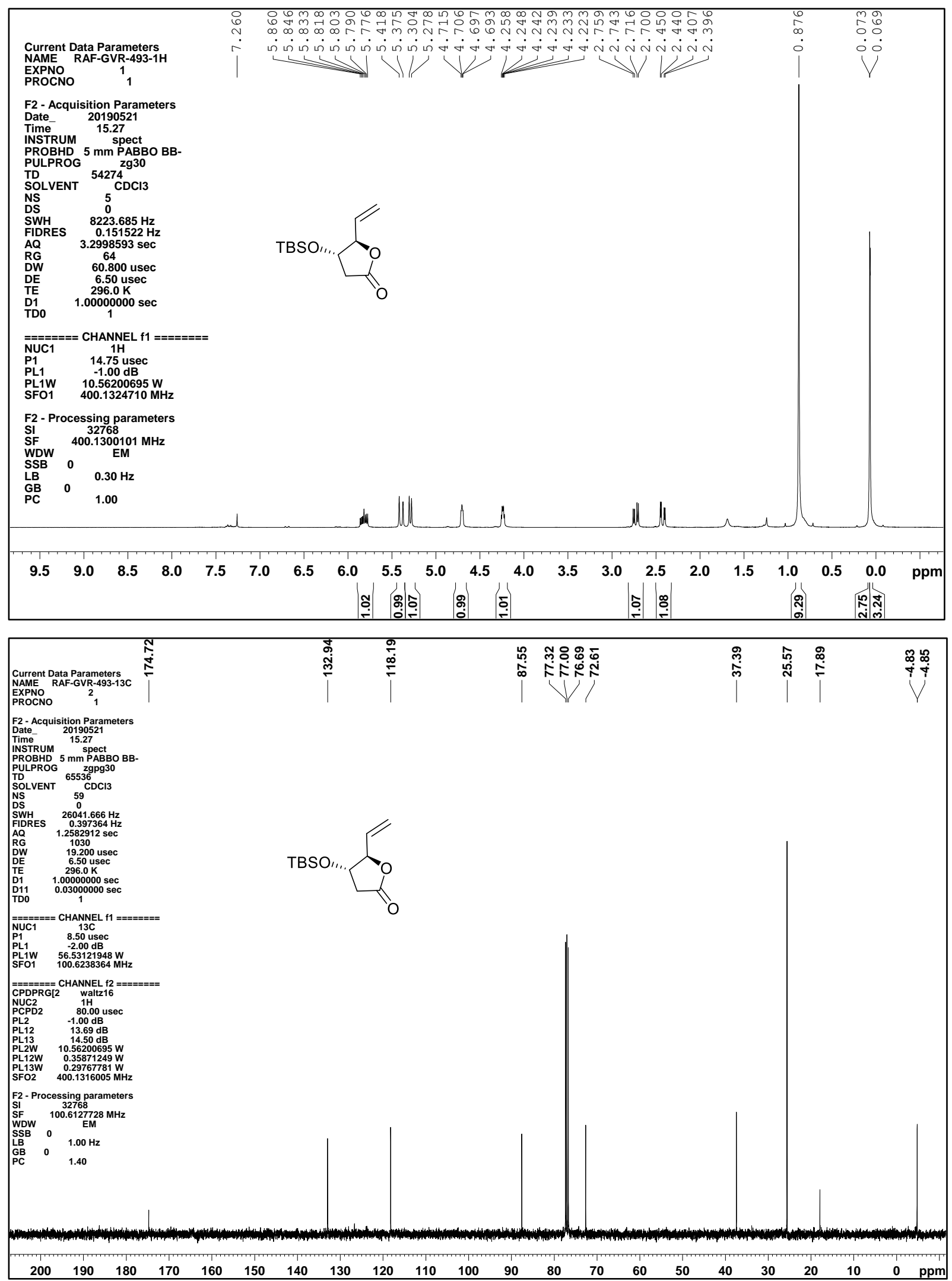
${ }^{1} \mathrm{H}$ NMR $\left(500 \mathrm{MHz}, \mathrm{CDCl}_{3}\right)$ and ${ }^{13} \mathrm{C}$ NMR $\left(125 \mathrm{MHz}, \mathrm{CDCl}_{3}\right)$ of compound 24
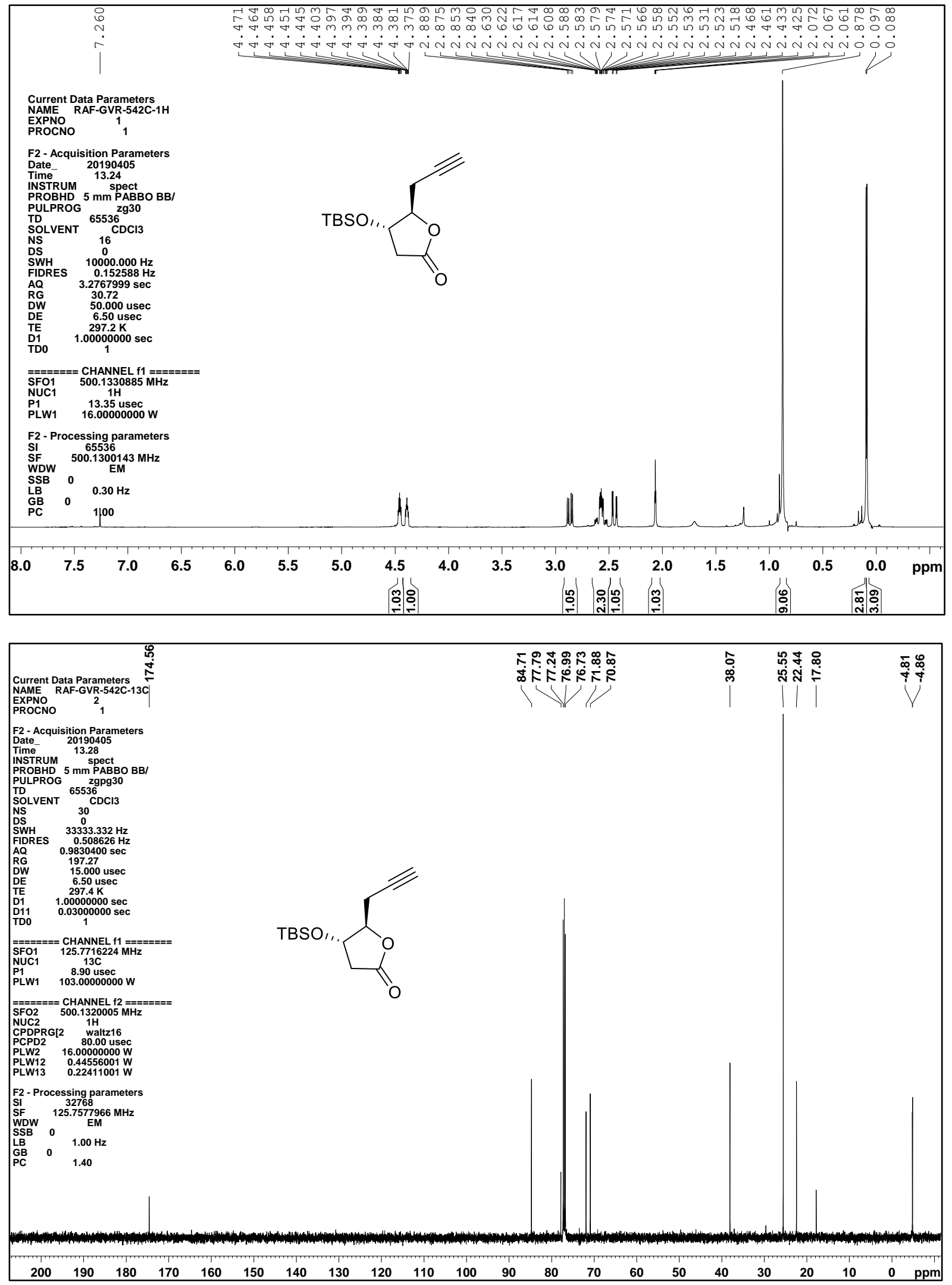
${ }^{1} \mathrm{H}$ NMR $\left(500 \mathrm{MHz}, \mathrm{CDCl}_{3}\right)$ and ${ }^{13} \mathrm{C}$ NMR $\left(125 \mathrm{MHz}, \mathrm{CDCl}_{3}\right)$ of compound 25
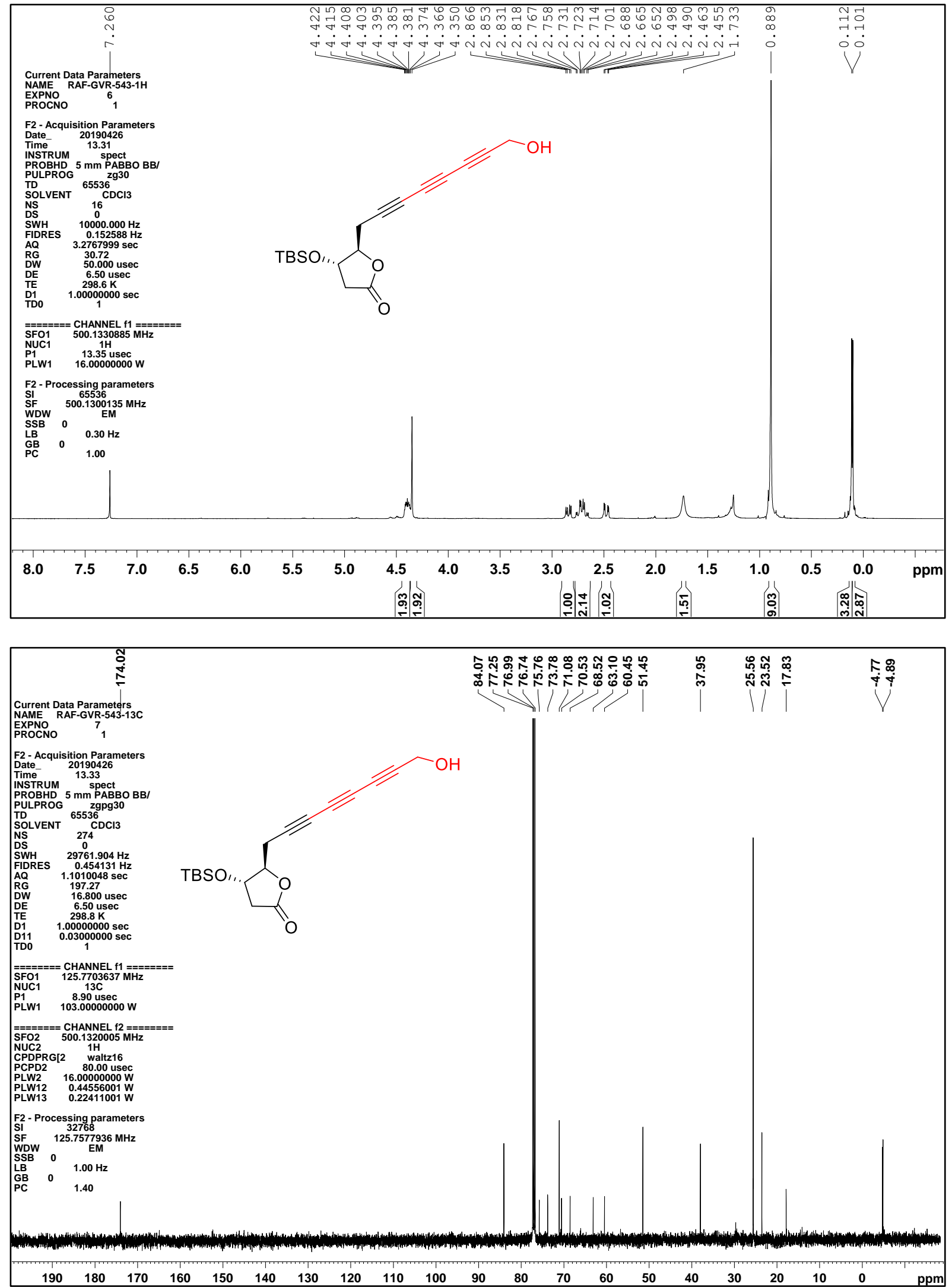
${ }^{1} \mathrm{H}$ NMR (500 MHz, acetone $d_{6}$ ) of compound 1a and known spectra

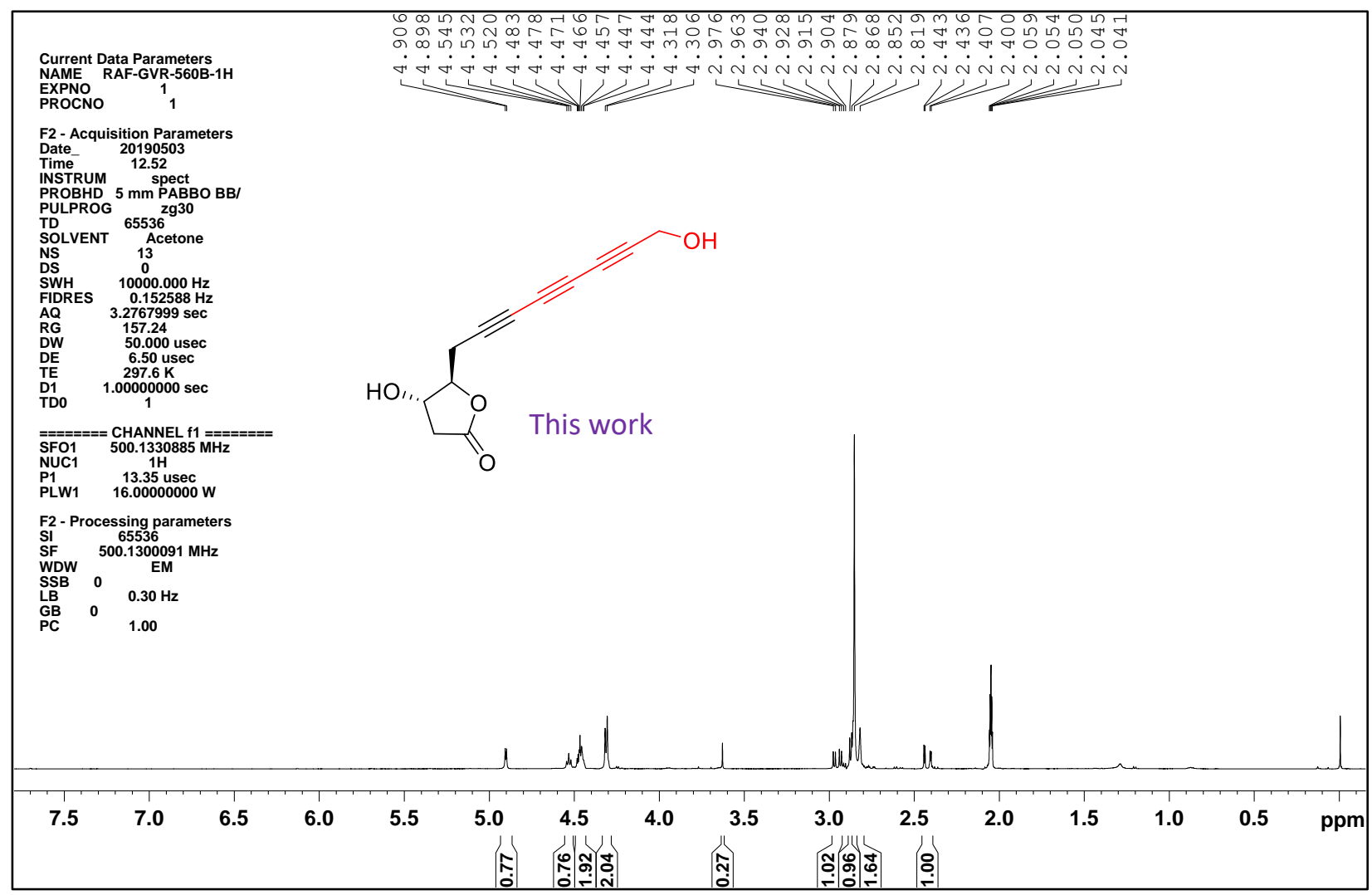

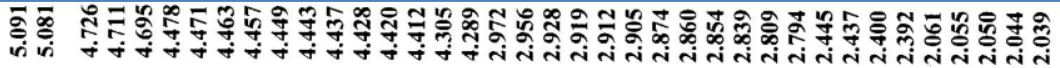

Synthesis by Mohapatra, Org. Lett. 2017, 19, 4167

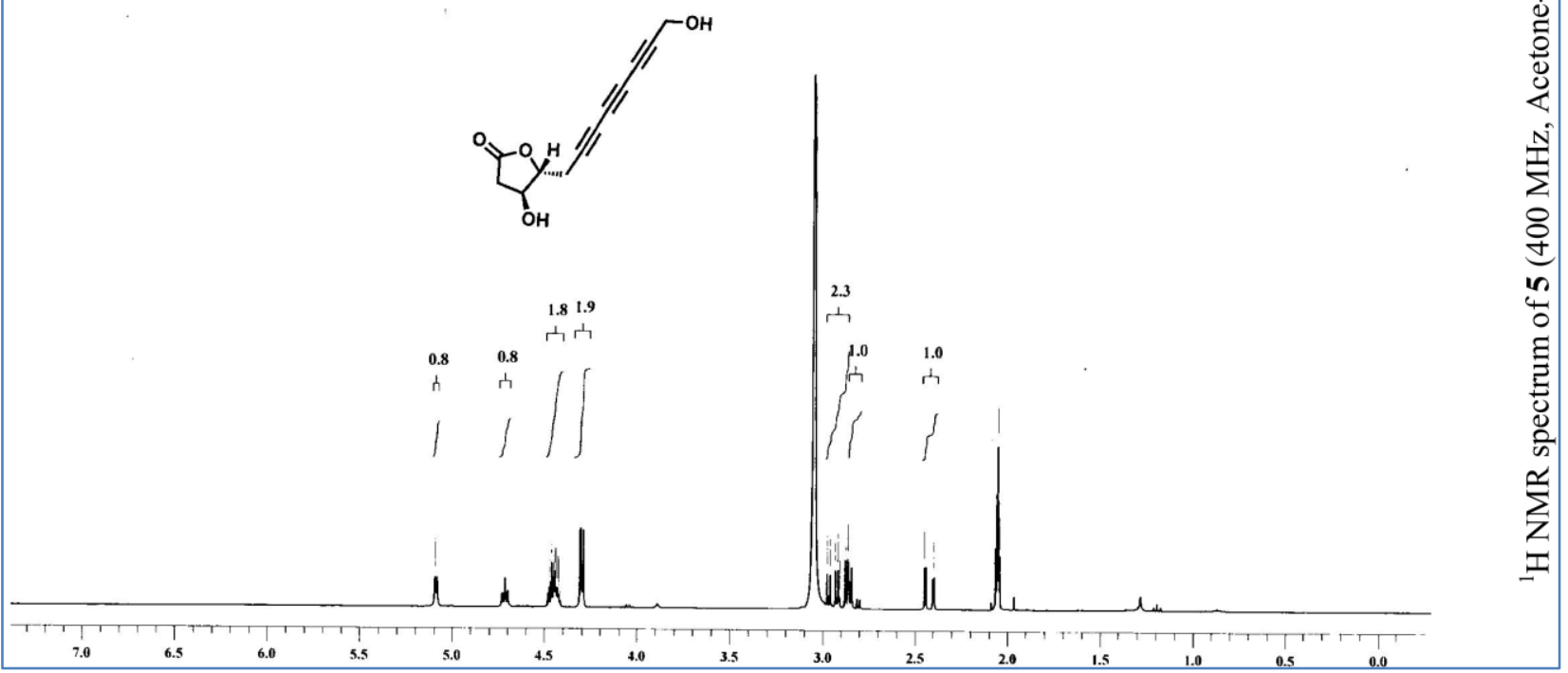


${ }^{1} \mathrm{H}$ NMR (400 MHz, acetone $d_{6}+\mathrm{D}_{2} \mathrm{O}$ ) of compound 1a. The peaks between $\delta 4.50-5.00$ in the ${ }^{1} \mathrm{H}$ NMR of 1a on Page S23 were that of OH protons.

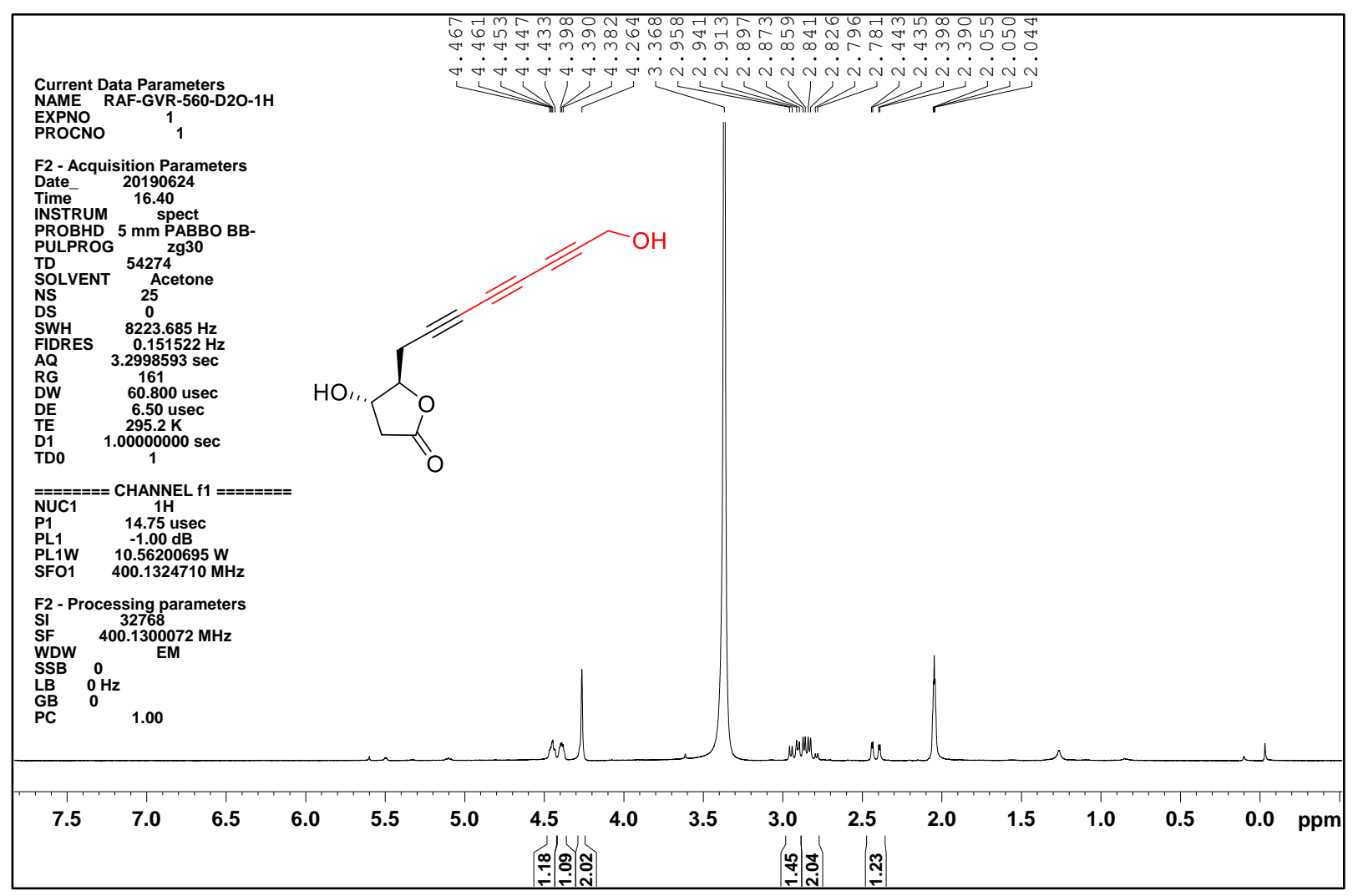


${ }^{13} \mathrm{C}$ NMR (125 MHz, acetone $d_{6}$ ) of compound 1a and known spectra
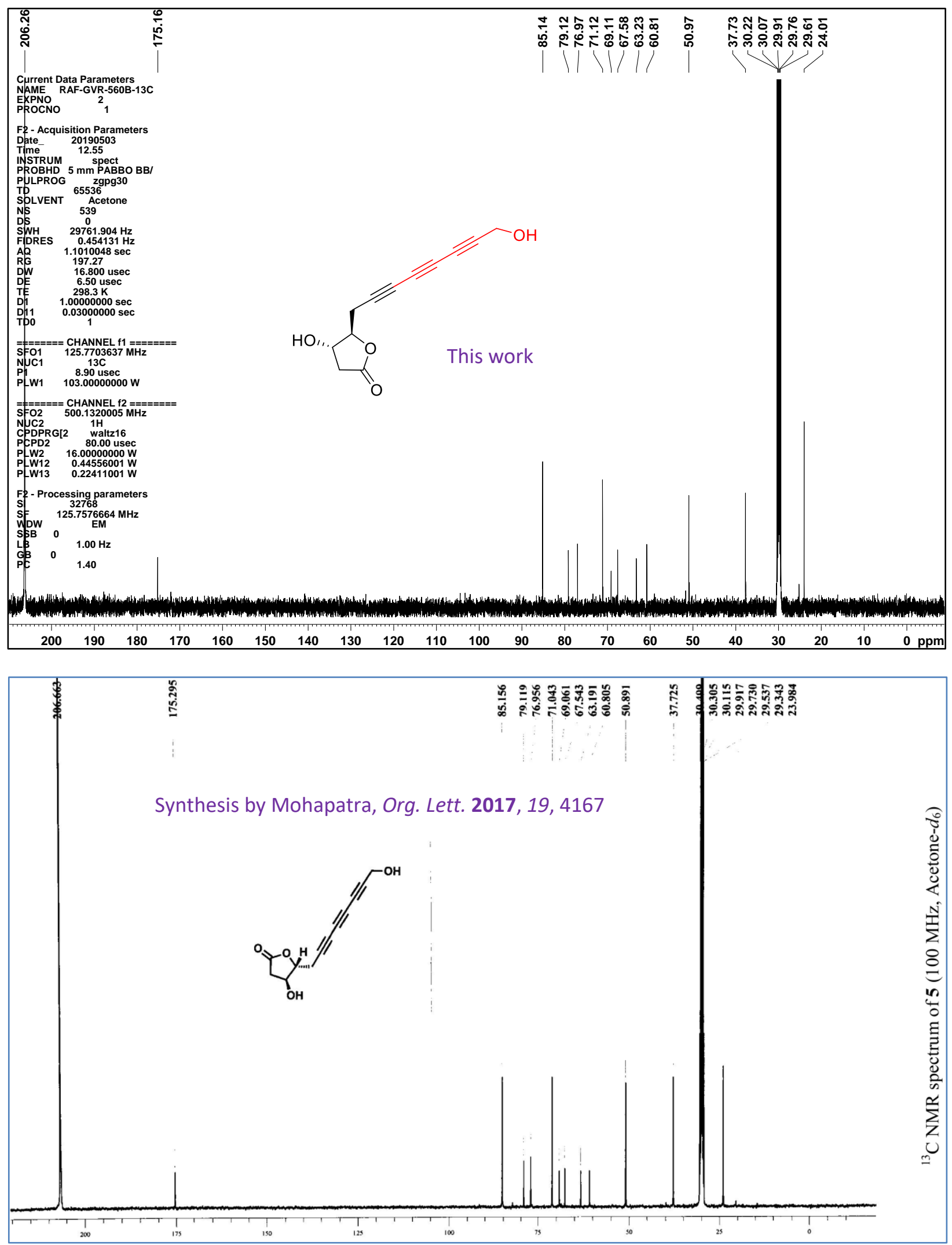